\title{
Stephanoscyphus (Scyphozoa, Coronatae) und seine direkte Abstammung von den fossilen Conulata ${ }^{1}$
}

\author{
BERNHARD WERNER \\ Biologische Anstalt Helgoland, Zentrale, Hamburg
}

\begin{abstract}
Stephanoscyphus (Scyphozoa, Coronatae) and its direct evolution from the fossil Conulata. The scyphopolyp Stephanoscyphus Allman 1874 represents the polyp generation of the scyphomedusan order Coronatae. Though this polyp has been known for more than a hundred years its general morphology, systematics, and evolution have been inadequately described. Participating in the International Indian Ocean Expedition, 1964 to 1965, on board of the German research vessel "Meteor", the author was able to collect a sufficient supply of living Stephanoscyphus off the coasts of South Arabia and East Africa. For the first time, it was possible to rear these polyps in laboratory cultures. A thorough investigation of morphology, developmental history and behaviour based on longterm observations of the living polyps gave clear indications that Stephanoscyphus directly descended from the fossil group of Conulata, the scyphozoan nature of which has been affirmed by KIDERLEN (1937) and KNIGHT (1937). The main feature which Stephanoscyphus has in common with the Conulata is the possession of a periderm tube. The characteristics found in a detailed investigation of the periderm tube conform well with those found in the periderm of the Conulata except for the closure of the aperture by triangular flaps which are absent in Stephanoscypisus. The soft body contains primitive features as well. Hence it must be concluded finally that the type of organization which the fossil ancestors exhibited has survived in Stepbanoscypbus and that the Coronatae represent the most basic group of all living Scyphozoa. On the other hand, the results give strong support for the scyphozoan nature of the Conulata, the organization and life history of which have been elucidated by the observations of the living representatives of Stephanoscypbus.
\end{abstract}

\section{EINLEITUNG}

Die Erforschung der Stammesgeschichte der wirbellosen Tiere wird bei vielen niederen Gruppen dadurch erschwert, daß keine oder nur wenige Uberreste ihrer Vorfahren aus vergangenen geologischen Epochen erhalten geblieben oder entdeckt sind, Daher ist unsere Kenntnis der Phylogenie der niederen Wirbellosen auch heute noch mit zahlreichen Unsicherheiten behaftet. Bei vielen Gruppen bleibt nur die Möglichkeit, aus der vergleichenden Betrachtung der Morphologie und Lebensweise heute lebender Tierformen auf die Organisation ihrer Vorfahren zu schließen und auf diese Weise zu versuchen, die Lücken der stammesgeschichtlichen Zusammenhänge zu schließen.

1 Erweiterter Kurzportrag, der am 29. September 1966 unter dem Titel „Stephanoscypbus (Scyphozoa Coronatae), ein rezenter Vertreter der Conulata?" auf der Jahresversammlung der Paläontologischen Gesellschaft in Münster i. W. gehalten wurde. 
Unter diesem Aspekt haben vergleichende morphologische und entwidklungsgeschichtliche Untersuchungen eine stets aktuelle Bedeutung. Das triff in besonderer Weise für Tierformen zu, die entweder neu entdedst werden oder die zwar bekannt, aber aus irgendwelchen Gründen nur unzulänglich untersucht sind. Ein Musterbeispiel für den letzteren. Fall ist der Scyphopolyp Stephanoscyphus, der schon vor über 100 Jahren aufgefunden und von Aliman (1874) nach Material aus dem Mittelmeer zuerst als eigene Gattung beschrieben, aber irrtümlich in die Hydrozoa eingereiht wurde. Lo Bianco \& Mayer (1890) entdeckten die ungeschlechtliche Fortpflanzung des Polypen durch Strobilation, womit die eindeutige Zugehörigkeit zu den Scyphozoa erwiesen war. Form und Entwicklungszustand der vom Polypen erzeugten Jungmedusen ließen keinen $Z$ weifel daran zu, daß Stephanoscyphus die Polypengeneration der CoronatenMedusen darstellt.

Polypen des nicht eben häufigen, aber auch nicht seltenen Genus Stephanoscyphus wurden seitdem auf dem Schelf fast aller Kontinente wie auch in größeren Meerestiefen gefunden (Leloup 1937, Kramp 1959, Naumov 1959, Moore 1961, Brahm \& Gerger 1966). Wenn trotzdem Biologie und Entwicklungsgeschichte weitgehend unbekannt geblieben sind, so ist die Ursache darin zu suchen, daß der Polyp nur sehr selten lebend beobachtet wurde, und daß sich das konservierte Material durchweg in einem ungenügenden Erhaltungszustand befand. Der einzige neuere Autor, der lebende Polypen für seine Untersuchungen verwendet hat, ist Komar (1935). Doch handelt es sich bei der von ihm untersuchten Art $S$. racemosus um eine Litoralform, deren Lebensgeschichte und Bau in mehrfacher Hinsicht als spezialisiert bezeichnet werden müssen, so daß die Ergebnisse nicht verallgemeinert werden können. Nach allen älteren Untersuchungen kann als gesicherte Tatsache nur gelten, daß die Arten der Gattung Stephanoscyphus Coronaten-Medusen erzeugen. Diese Medusen sind vor allem in den Tropen weitverbreitet und leben zum Teil in der Tiefsee.

Als Teilnehmer der internationalen Expedition in den Indischen Ozean 1964 bis 1965 mit dem deutschen Forschungsschiff „Meteor" fand ich in dem Dredge-Material mehrerer Stationen vor der südarabischen und ostafrikanischen Küste zahlreiche lebende Stephanoscyphus, die zunächst an Bord gehältert und später auf der Heimreise lebend mirgenommen werden konnten. So war es erstmals möglich, diese Form in Laboratoriumsdauerkultur zu nehmen und eingehende Beobachtungen über Organisation, Lebensweise und Entwicklungsgeschichte anzustellen.

Die Ergebnisse der bisherigen Untersuchungen wurden in einem ersten Beitrag (WERNER 1967, im Druck) mitgeteilt. Dabei wurde auch schon kurz auf die Bedeutung von Stephanoscypbus für die Evolution der Scyphozoa und auf seine Abstammung von den Conulata hingewiesen. Diese ausgestorbene Tiergruppe ist seit langem durch zahlreiche Fossilfunde wohl bekannt; aber erst KIDERLEN (1937) und KNIGHT (1937) ist es gelungen, die bestehenden. Unsicherheiten der systematischen Einordnung weitgehend zu beseitigen und ihre Scyphozoennatur zu begründen. Wenn danach die Conulata als die Vorfahren aller rezenten Scyphozoen angesehen werden müssen, so triff dies allgemein auch für Stephanoscyphus zu.

Die weitere Prüfung der stammesgeschichtlichen Zusammenhänge und ein eingehender Vergleich der strukturellen Einzelheiten aber ergab, daß Stephanoscyphus überraschend große Ahnlichkeiten in Bau und Lebensweise mit den nur durch Fossilfunde 
bekannten Conulata hat. Dementsprechend ist das Ziel der vorliegenden Arbeit nachzuweisen, daß Stephanoscyphus als direkter Nachkomme dieser ausgestorbenen Vorfahren gelten darf. Umgekehrt läßt sich auf diese Weise zeigen, daß wir in Stephanoscyphus den lebenden untrüglichen Beweis für die Scyphozoennatur der Conulata vor uns haben. Diese Theorie wird zwar meist anerkannt und ist in die modernen paläontologischen Lehrbücher eingegangen, wurde aber von TERMIER \& TERMIER (1948) erneut ernsthaft bezweifelt. Schließlich versetzt uns die Kenntnis von Stephanoscypbus in die Lage, Rückschlüsse auf die Organisation und Lebensweise seiner fossilen Vorfahren zu ziehen. Die Beantwortung der Frage nach dem historischen Informationsgehalt von Stephanoscyphus kann daher besser begründete Vorstellungen über die Lebensgeschichte und darüber hinaus auch über die Ursachen des Aussterbens der Conulata liefern, als sie auf der Grundlage der bisherigen Kenntnisse möglich waren.

\section{BAU UND SCYPHOZOENNATUR DER CONULATA}

Die ausgestorbenen Conulata sind durch die zahlreichen Fossilfunde ihrer Gehäuse gut bekannt. Eingehende Beschreibungen ihres Baues, ihrer Systematik und Lebensweise haben KIDERLEN (1937), TERMIER \& Termier (1948) gegeben (vgl. ferner BoučeK 1939, Moore \& Harrington 1956, Müller 1963; bei diesen Autoren weitere Literatur). Daher genügt es, die wesentlichen Tatsachen in einer tabellarischen Ubersicht zusammenzustellen, die den Vergleich mit Stephanoscypbus erleichtern kann.

Klasse Scyphozoa, Unterklasse Conulata, einzige Ordnung Conulariida, 4 Familien mit 20 Gattungen und ca. 200 Arten, von denen manche mit Tausenden von Funden vertreten sind; marin; Kambrium bis zur Trias.

Form: Überwiegend schlanke pyramidenförmige, seltener hochkonische oder breitkonische, sehr dünnwandige und biegsame Gehäuse, gerade oder schwach gebogen. Jugendstadien am geschlossenen Apikalende mit einer kleinen Haftscheibe angewachsen. Alte Tiere meist abgelöst; ihr Basalende mit einer sekundär gebildeten Querwand (Schott, Diaphragma) verschlossen. Zuweilen mehrere Diaphragmen am unteren Ende kammerartig übereinander angeordnet. Mündung bei den pyramidenförmigen Gehäusen mit vier dreieckigen Klappen verschlossen. Gehäuse im Querschnitt von tetramerer Symmetrie.

Oberflä chenst r uktur: Seitenflächen der pyramidenförmigen Gehäuse of mit Mittel- und Nebenlinien. Kanten und Mittellinien können auf der Innenseite leistenförmige Verstärkungen aufweisen. Außere Oberfläche meist mit Quer- und Längsstreifen bedeckt, Innenseite glatt.

Chem is che Natur: Chitin oder Chitin-Calciumphosphat.

Farbe : Grundfarbe dunkelbraun, weitere Färbungen von rötlichbraun, violettbraun bis blaugrau, lichtgrau, weiß.

Größ e : Meist 40 bis $100 \mathrm{~mm}$, einzelne Arten bis zu $400 \mathrm{~mm}$.

Dicke der Gehäusew andung: Maximal $1,0 \mathrm{~mm}$, meist nur Bruchteile eines mm. Genaue Angaben finden sich bei KrDERLEN (1937, p. 118) für folgende Arten: Conularia imperialis 0,056 bis $0,07 \mathrm{~mm} ; 0,32 \mathrm{~mm} ; 0,4$ bis $0,5 \mathrm{~mm}$; C. exquisita 
0,17 bis $0,28 \mathrm{~mm}$; C. fecunda 0,068 bis $0,766 \mathrm{~mm} ; 0,170$ bis $0,735 \mathrm{~mm}$; C. stromeri 0,1 bis $0,3 \mathrm{~mm}$.

Durchmesser am Basalteil über der Haftplate: $0,25 \mathrm{~mm}$ bei C. gracilis nach KIDERLEN (p. 134).

A ufbau der Gehäusewandung: Aus mehreren Schichten, die in sich fein geschichtet sind.

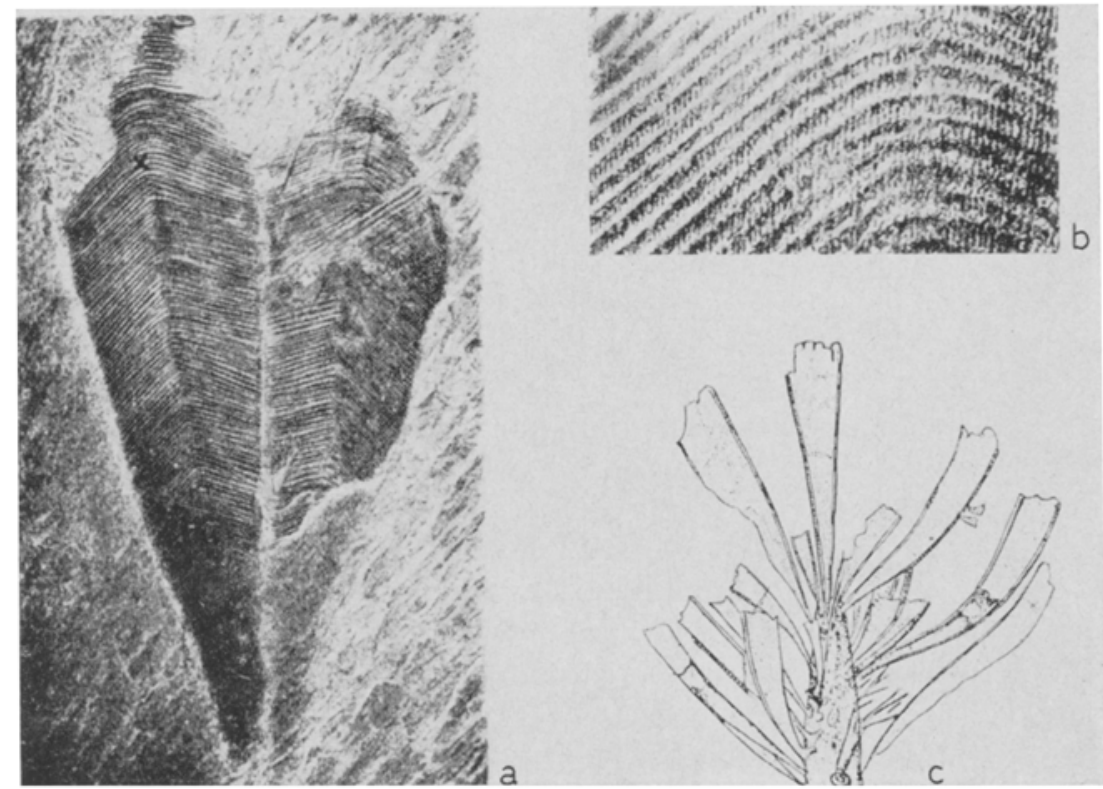

Abb. 1 a-c: Bau und Oberflächenstruktur der Conulata. a, b Conularia gemündina RuD. RICHTER, a Seitenansicht, b Teilstück mit Quer- und Längsstreifung der Oberfläche. c Sphenothallus angustifolius HaLL, Jungtiere, die mit Haftscheiben auf älteren angeheftet sind. $(\mathrm{a}, \mathrm{b}$ nach Richter \& Richter 1930, c nach RuEdemanN 1898, aus Müller 1963, p. 162, Fig. 176, 177)

Lebensweise: Uberwiegend solitär; in der Juged angeheftet, im erwachsenen Zustand abgelöst und passiv vagil; zu der von KIDERLEN vermuteten Möglichkeit einer freischwimmenden Lebensweise nach Art einer Meduse siehe p. 343-344. Die Abbildungen 1,2 sind geeignet, die tabellarische Übersicht zu illustrieren und überdies einen Eindruck davon zu vermitteln, wie man sich die lebenden Conulata nach KIDERLEN (1937) yorzustellen hat.

Als Beweismittel für die Begründung der Scyphozoennatur der Conulata hat KIDERLEN die damals bekannten Tatsachen über Bau und Struktur der rezenten Scyphozoen verwendet, wobei er vor allem auf die für diese Klasse charakteristische tetraradiale Symmetrie des Körperbaues hinweisen konnte. Bei dem als Organisationstypus wohlbekannten, oft untersuchten und abgebildeten Scyphistoma, dem Polypen der Ordnungen Semaeostomeae und Rhizostomeae, der nur wenige $\mathrm{mm}$ groß ist und bis heute als Grundform des Scyphopolypen angesehen wird (Chapman 1966, Thiel 
1966), kommt die tetraradiale Organisation in der Kreuzform der Mundlippen, im Vorhandensein der vier Gastralsepten bzw. Gastraltaschen, der vier Septaltrichter und der vier ektodermalen Septalmuskeln klar zum Ausdruck.

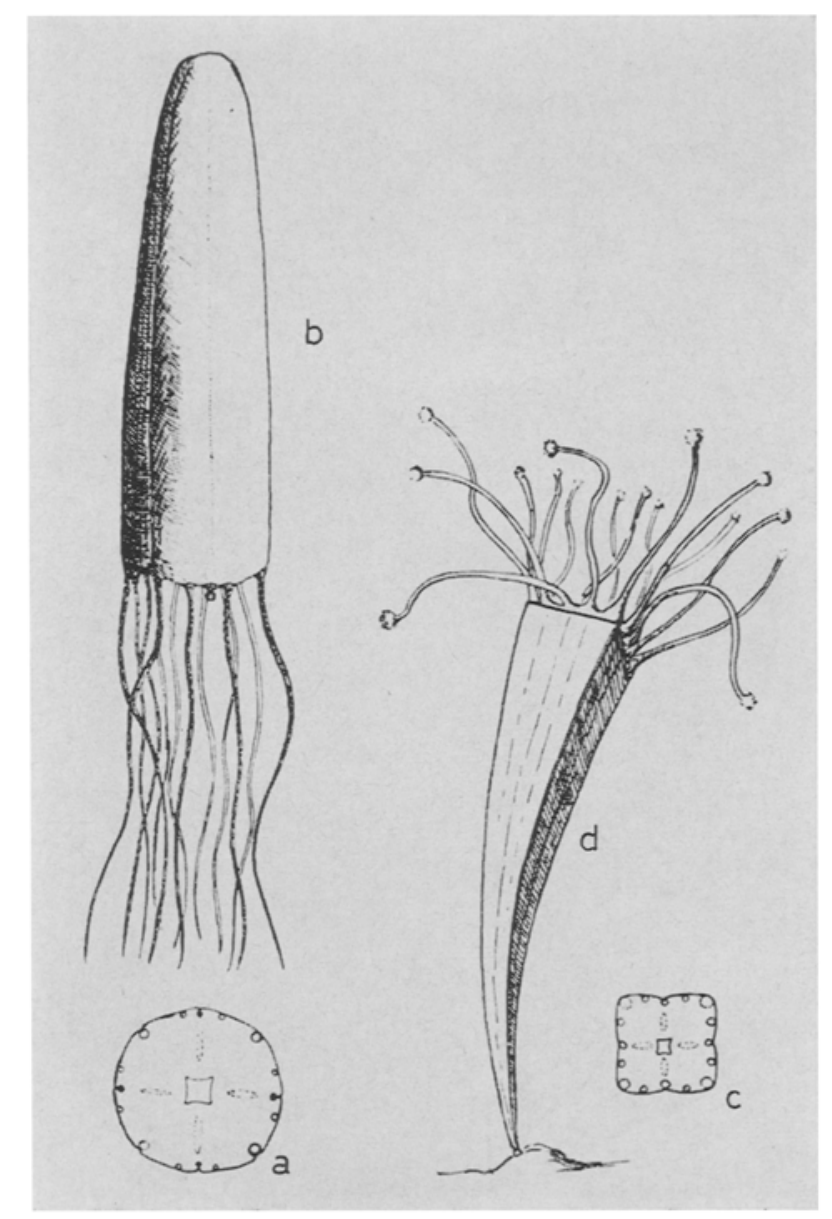

Abb. 2 a-d: Rekonstruktionen von Conularien, a, b Exoconularia consobrina (BARRANDE); a Querschnitt, b Rekonstruktion als schwimmende Meduse. c, d Archaeocontilaria fecunda (BARRANDe), c Querschnitt, d Rekonstruktion. (Nach KIDERLEn 1937, aus Müller 1963, p. 163, Fig. 178, 179)

Umgekehrt konnte KIDERLEN zeigen, daß manche vorher schwer verständlichen Besonderheiten des Körperbaues der rezenten Scyphozoen einer einfachen Erklärung offenstehen, wenn sie als altes Erbteil gedeutet werden, das sie von den fossilen Vorfahren übernommen und bis heute bewahrt haben. Ein auffallendes Beispiel ist mit dem Vorhandensein und der ektodermalen Natur der vier Septalmuskeln beim Scyphopolypen gegeben, das in übeteinstimmender Weise auch bei den Stauromedusen vor- 
kommt, die polypoide und medusoide Merkmale in sich vereinigen. Vom rein funktionellen Standpunkt aus betrachtet, läßt sich kaum eine plausible Erklärung für Existenz und Eigenart dieses anatomischen Merkmals geben. Leitet man aber die vier interradialen Septalmuskeln von den bei den Conulata zu postulierenden Muskeln ab, die an den vom Ektoderm erzeugten Mündungsklappen ansetzten und deren Verschluß bewirkten, so werden Vorhandensein und rein ektodermale Natur der genannten Muskeln beim rezenten Scyphopolypen auf der angedeuteten historischen Grundlage ohne weiteres verständlich (vgl. dazu auch CHAPMAN 1966, p. 56-60).

\section{ORGANISATION, LEBENSWEISE UND ENTWICKLUNGSGESCHICHTE VON STEPHANOSCYPHUS}

In den meisten Beschreibungen und Abbildungen des Scyphistoma fehlt jeglicher Hinweis auf das Vorhandensein peridermaler Bildungen; doch hat bereits Claus (1883, vgl. Kiderlen 1937, Chapman 1966) die Existenz eines feinen Peridermhäut-

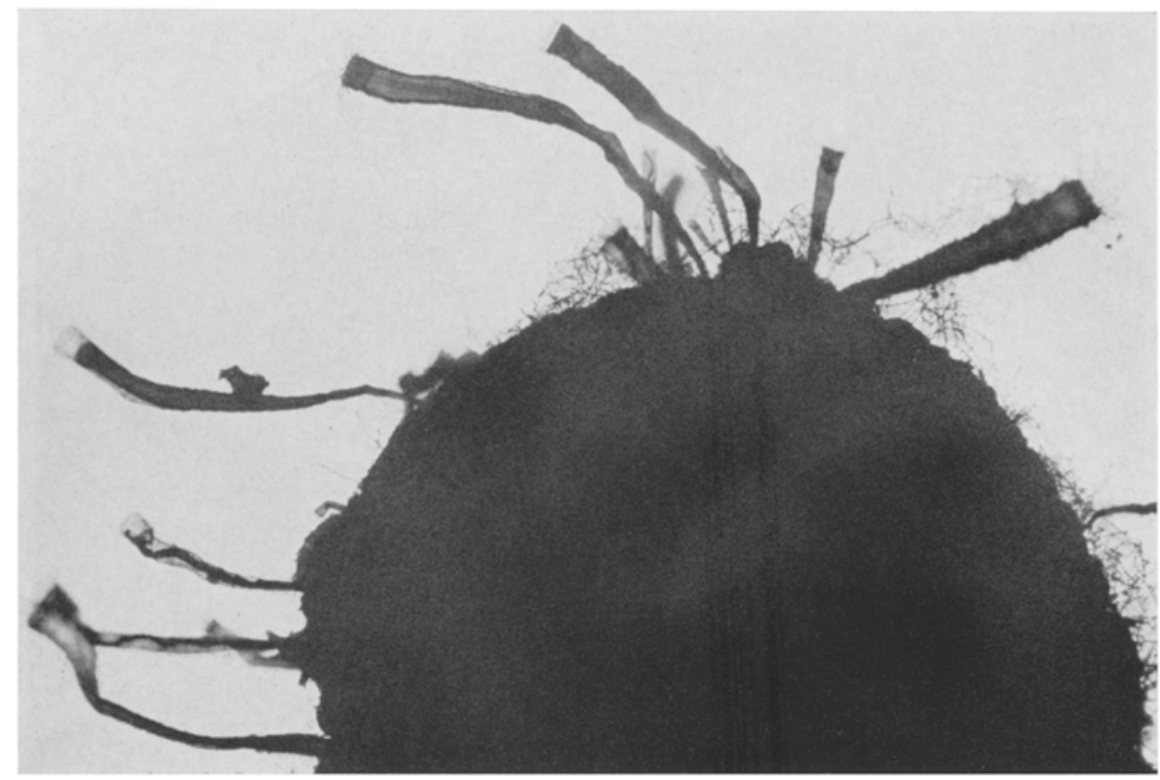

Abb. 3: Stephanoscyphus in natürlicher Lebensstellung, zahlreiche Exemplare an einer Muschelschale angeheftet

chens nachweisen können, das die Polypenbasis umgibt. Es ist allerdings sehr zart und entzieht sich aus diesem Grunde meist der unmittelbaren Beobachtung. Im Gegensatz dazu ist Stephanoscyphus durch den Besitz einer festen, den Polypen vollständig umgebenden Peridermröhre ausgezeichnet, durch die er sich von sämtlichen rezenten Scyphopolypen unterscheidet und der er seine besondere 
Bedeutung verdankt. Denn es ist gerade dieses Merkmal, das durch sein Vorhandensein ebenso wie durch die strukturellen Details den Anschluß von Stephanoscypbus an die Conulata primär ermöglicht. Angesichts der Tatsache, daß von den fossilen Vorfahren nur sehr spärliche Überlieferungen über den Bau des Weichkörpers erhalten sind, muß sich entsprechend der Beweis des behaupteten direkten Zusammenhanges auf die Prüfung von Bau und Eigenschaften der Peridermröhre bei Stephanoscyphus konzentrieren.

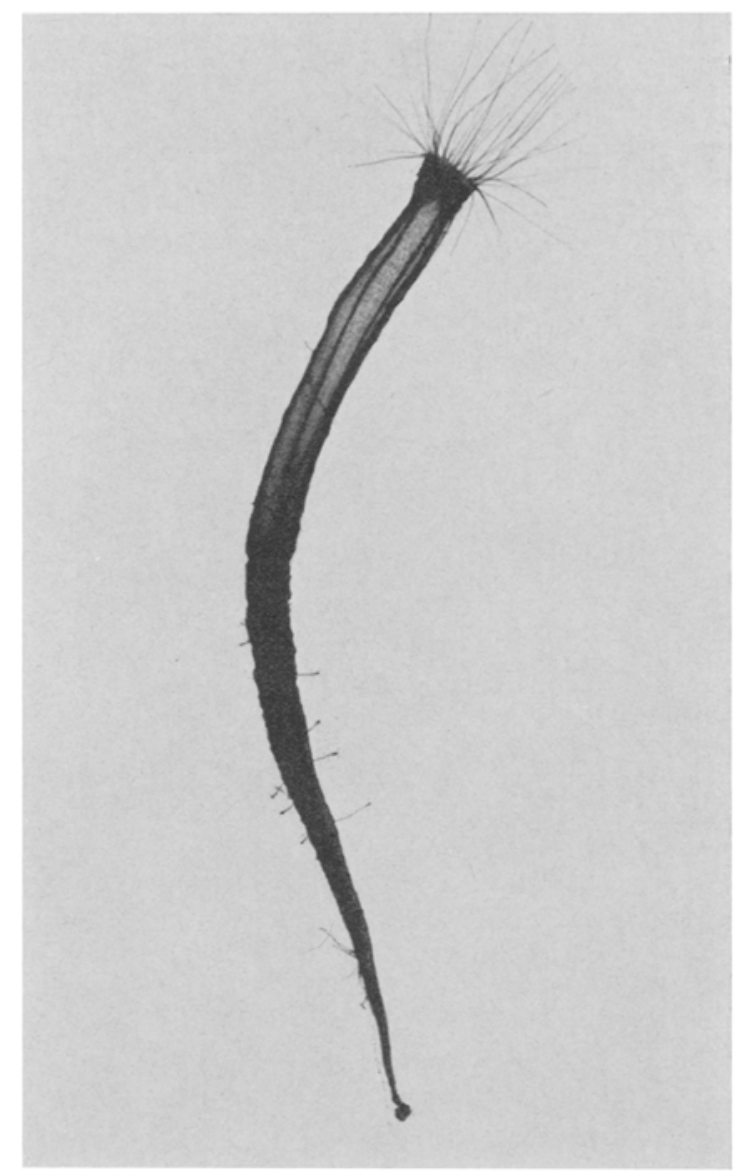

Abb. 4: Stephanoscyphus, Lebendphoto des vom Ansatzkörper abgelösten Polypen der Coronatenmeduse Nausithoë punctata KölLIKER mit ausgestrecktem Kopfteil und Tentakelkrone. Die Haftscheibe am Basalteil ist erhalten

Der Polyp ist solitär und während seines ganzen Lebens mit einer kleinen Haftscheibe an einem Ansatzkörper, einer Molluskenschale oder einem Stein, befestigt. Auch nach seinem Tod bleibt die leere Röhre angeheftet. Eine Muschelschale mit einer ganzen Anzahl lebender Polypen, die im Dredge-Material vor der südarabischen Küste gefunden wurde, ist in Abbildung 3 dargestellt. 
Das Bild eines vom Ansatzkörpers abgelösten Polypen (Abb. 4) ist gut geeignet, seinen allgemeinen Habitus zu demonstrieren. Der Weichkörper ist in ganzer Länge von der Peridermröhre umgeben, aus deren Mündung oben nur der Kopfteil mit einem Kranz von zahlreichen Tentakeln herausschaut. Durch die im oberen Teil sehr feine Röhrenwandung sind die vier Gastralsepten des Weichkörpers deutlich zu erkennen. In der unteren Hälfte ist die Wandung der Röhre verdickt und daher undurchsichtig. Hier ist die äußere Oberfläche bei dem abgebildeten Exemplar mit einem koloniebildenden Hydroidpolypen bewachsen. Das ist deswegen erwähnenswert, weil die Außenseite des Gehäuses der Conulata ebenfalls tierischen Bewuchs aufweisen konnte, beispielsweise von Echinodermen, Bryozoen, Brachiopoden.

\section{Die Peridermröhre}

Die Einzelheiten über Form und Struktur des Gehäuses sind zum Vergleich in der folgenden Ubersicht zusammengestellt:

Fo r m : Schlank konische, düinnwandige und biegsame Gehäuse mit kreisrundem Querschnitt. Basalteil mit einer kleinen rundlichen, meist unregelmäßig gelappten Haftscheibe angewachsen. Röhre mehr oder weniger gerade bis schwach hornförmig gebogen.

Oberflächenstruktur: Außere Oberfläche mit einem regelmäßigen Muster aus Quer- und Längsstreifen bedeckt, innere Oberfläche glatt. Auf der Innenseite befinden sich im Basalteil mehrere übereinanderliegende, in einem Kranz angeordnete, zahnähnliche Bildungen von je vier großen und vier kleinen Zähnen, die sich kreuzweise gegenüberstehen.

Chemische Natur: Chitin (nach ChapMaN 1966, p. 56).

Fa rbe: Grundfarbe braun, von dunkelbraun im Basalteil mit verdickter Wandung bis hellbraun oder hellgelb an der oberen Mündung.

Größe : Meist 10 bis $20 \mathrm{~mm}$, bis zu maximal $56 \mathrm{~mm}$.

Dicke der Gehäusew andung: basal 0,015 bis $0,030 \mathrm{~mm}$, an der Mündung 0,005 bis $0,006 \mathrm{~mm}$.

Durchmesser der $M$ ündung: 0,5 bis $3,0 \mathrm{~mm}$.

Durchmesser a $\mathrm{B}$ as a 1 te il über der Haftplatte: 0,15 bis $0,3 \mathrm{~mm}$.

A u fbau der Gehäusewandung: Aus zwei Hauptschichten, die in sich fein geschichtet sind.

Diese kurze Zusammenstellung der strukturellen Details läßt erkennen, daß eine weitgehende Ahnlichkeit der Peridermröhre von Stephanoscyphus mit dem Gehäuse der Conulata besteht und daß sie sich diesem durch Größe, Farbe, Oberflächenstruktur, chemische Natur, Aufbau der Gehäusewand unmittelbar anschließt. Ein wichtiges Merkmal ist die Oberflächenstruktur, die aus einem im Normalfall des ungestörten Wachstums sehr regelmäßigen Muster aus Quer- und Längsstreifen besteht. Die nähere Untersuchung am lebenden Tier konnte die Entstehung dieses Musters der Oberflächenstruktur aufklären. Die Querstreifen, oder besser gesagt, die Querringe - da der Röhrenquerschnitt ja rund ist - entstehen als Folge einer periodischen, diskontinuierlichen Bildungsweise der Röhre. Diese wird von den Drüsenzellen in der Außen- 
epidermis des aus der Röhre herausgestreckten und voll entfalteten Kopfteils erzeugt. In diesem Zustand hat die Außenseite des Kopfteils außerhalb der Röhre die Form eines Kragens ( $\mathrm{kr}$, Abb. 9a), dessen Epidermis zahlreiche Drüsenzellen trägt. Am oberen Rand des Kragens, der bei einem Tier einer Gesamtlänge von 19,0 mm eine Höhe von $0,5 \mathrm{~mm}$ hat, inserieren die Tentakel, während sein unterer Rand von der

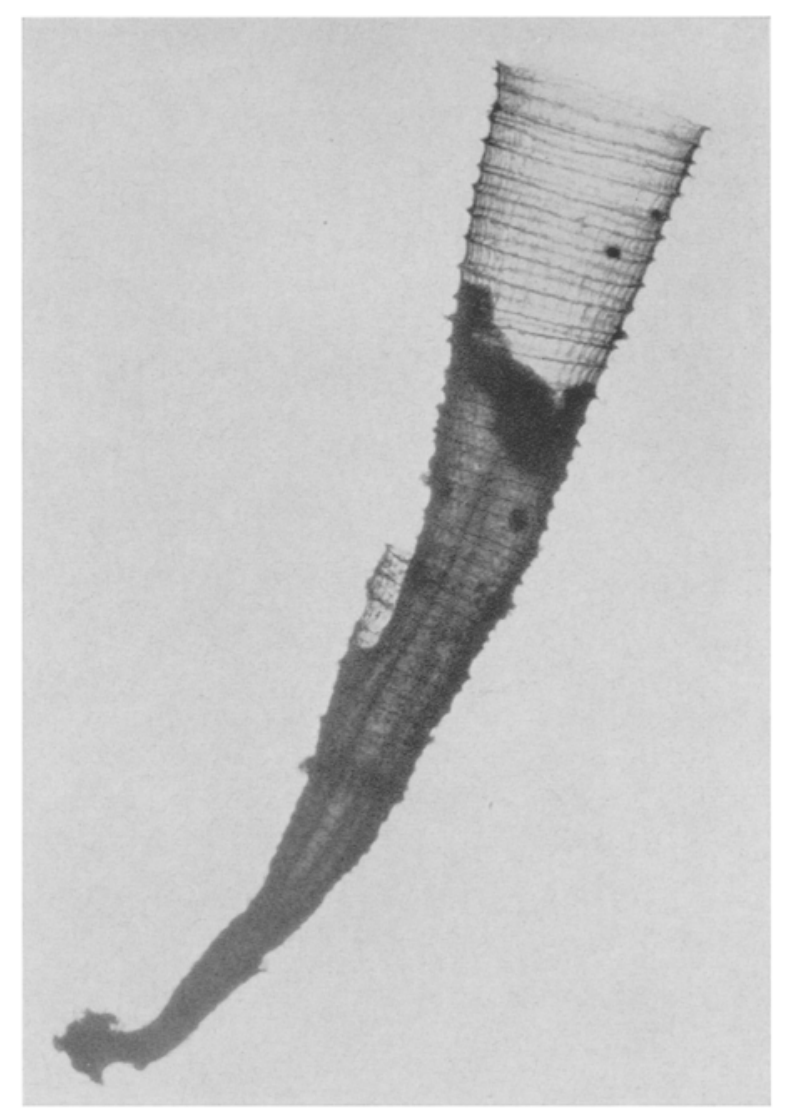

Abb. 5: Stephanoscyphus, Lebendphoto des in die Röhre zurückgezogenen Polypen der Coronatenmeduse Atorella vanhoeffeni BIGELOW, der nach Beendigung einer Strobilationsphase in Regeneration begriffen ist

Epidermis des Körpers, der von der Röhre umschlossen ist, durch eine feine Ringfalte abgesetzt ist. In dieser Ringfalte ist die innige Verbindung zwischen dem oberen Rand der Röhre und der Außenepidermis des Weichkörpers hergestellt, und hier ist der Ort, wo durch die Sekretionstätigkeit der Drüsenzellen neue Peridermsubstanz an den Mündungsrand der Röhre angefügt wird, wo also ihr Längenwachstum erfolgt. Jedesmal, wenn sich der Weichkörper durch eine äußere Reizung, beim Beutefang oder auch bei der Strobilation (s. p. 334) kontrahiert und in die Röhre zurückzieht (Abb. 5), wird die Verbindung zwischen dem oberen Rand der Röhre und der ektodermalen Bil- 
dungszone des Weichkörpers notwendig unterbrochen, so daß keine Röhrensubstanz ausgeschieden werden kann. Erst wenn sich der Körper des Polypen wieder ausstreckt und über den Rand der Mündung hinausragt, kann erneut die Bildung der Röhre einsetzen, und kann ein neuer Querring auf den vorhergehenden unteren aufgesetzt werden, so daß die Zahl der Querringe ein Ausdruck des von unten nach oben gerichteten periodischen Längenwachstums der Röhre und damit auch des Alters des 'Tieres ist.

Die Breite der Querringe und die Art, wie sie voneinander abgesetzt sind, stellen artspezifische Merkmale dar (vgl. p. 328 und Abb. 8 a-c); sie hängen überdies einmal vom Alter und Wachstum ab, so daß die Querringe in den basalen Teilen schmäler und weniger deutlich voneinander abgesetzt sind als im oralen Teil der Röhre. Andererseits wird die Breite der Querringe innerhalb gewisser Grenzen auch von Außeneinwirkungen beeinflußt und kann wechselnd oder unregelmäßig sein.

Die feine Längsstreifung der Querringe ist durch das Verteilungsmuster und die spezielle Tätigkeit der Drüsenzellen bedingt und prädisponiert, die im Außenepithel des Kragens liegen und durch ihre Sekretionstätigkeit die zunächst flüssige, dann im
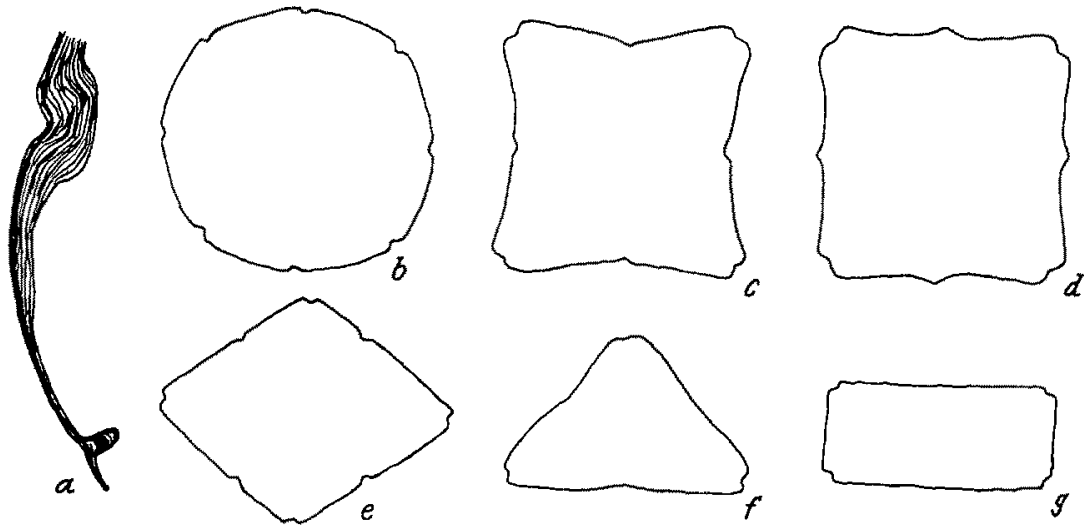

Abb. 6 a-g: Querschnitte durch Conulariengehäuse, aus BoučEк 1939, p. A 115, Fig. 2. a Teilquerschnitt zur Darstellung des Schichtaufbaues der Gehäusewandung; oben Kante, unten Mittellinie des Gehäuses

Seewasser erhärtende Röhrensubstanz ausscheiden. Damit ist die Bildungsweise der Oberflächenstruktur in ihren Grundzügen geklärt. Die Innenseite der Röhrenwandung ist glatt. Hier findet eine zwar recht langsame, aber doch stetige Verdickung der Wand statt, die von der Sekretionstätigkeit der Drüsenzellen ausgeht, die in der Außenepidermis des gesamten, von der Röhre umschlossenen Polypenkörpers liegen. Das führt im Endeffekt zu dem Aufbau der Gehäusewandung aus zwei Schichten, der äußeren, die die Strukturelemente des Oberflächenmusters trägt und sich in ihrer Dicke wenig verändert, und der inneren, die entsprechend ihrer Bildungsweise ohne Strukturelemente ist und ständig langsam an Dicke zunimmt. Die beiden Hauptschichten weisen in sich wiederum eine feine Schichtung auf, die aus der Tätigkeit der Drüsenzellen mit ihrer zeitlich aufeinanderfolgenden Ausscheidung der Peridermsubstanz gedeutet werden kann. 
Nach allem stimmt das Muster der Oberflächenstruktur der Röhre von Stephanoscyphus hinsichtlich der Quer- und Längsstreifung vollständig mit dem der Conulata überein. Und mehr noch, was bislang über die Entstehungsweise des Strukturmusters des Gehäuses der Conulata theoretisch erschlossen wurde (vgl. KIDERLEN 1937, p. 135, Termier \& Termier 1948, pp. 720-721), wissen wir bei Stephanoscyphus durch die direkte Beobachtung.

Ein Unterschied besteht im runden Querschnitt der Röhre. Da es aber auch Conulata mit elliptischem oder achteckigem bis rundlichem Querschnitt gegeben hat (vgl. Abb. 6), braucht man diesem Unterschied keine allzugroße Bedeutung beizulegen. Außerdem hatten auch die jungen Gehäuse der pyramidenförmigen Arten einen runden Querschnitt, der erst mit der Ausbildung des Klappenapparates quadratisch wurde (KIDERLEN, p. 135). Wichtige Unterscheidungsmerkmale sind allerdings das Fehlen
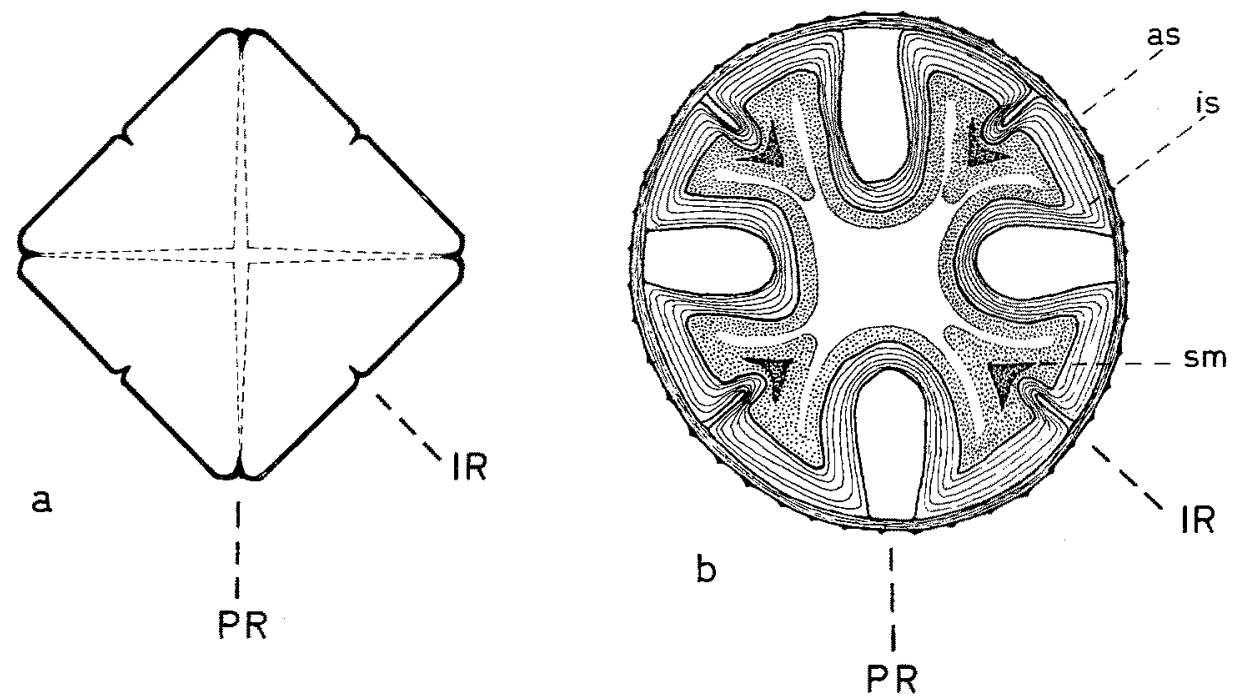

Abb. 7 a, b: Vergleich der Symmetrieverhältnisse und Peridermstrukturen der Conulata und des Stephanoscyphus (schematish). PR Perradialebene, IR Interradialebene. a Querschnitt durch ein Conulariengehäuse; die gestrichelten Linien sollen die Lage der Mündungsklappen andeuten; bei IR Mittellinie, bei PR Kante des Gehäuses. b Querschnitt durch einen Zahnkranz von Stephanoscyphus; bei IR kleiner Zahn, bei PR großer Zahn; as Außenschicht, is Innenschicht der Peridermwand; Weichkörper punktiert, sm Septalmuskel

eines aus dreieckigen Klappen bestehenden Deckelapparates, ferner von Längselementen, die den Kanten und Mittellinien des Gehäuses der Conulata entsprechen. Doch besitzt Stephanoscyphus seinerseits merkwürdige, abweichende Bildungen im Basalteil der Röhre. Es handelt sich um Peridermzähne auf der Innenseite der Röhre, die in Form von Kränzen übereinanderliegen und durch einen gewissen Abstand voneinander getrennt sind. Es sind meist 5 bis 7 Zahnkränze vorhanden, bei denen die sich entsprechenden Zähne genau in einer Vertikalreihe übereinanderliegen (vgl. die Seitenansichten der $A b b .13,14)$. Im Querschnitt ( $A b b .7 b$ ) sieht man, daß sie verschieden groß sind und daß sich immer vier größere und vier kleinere Zähne im Kreuz gegen- 
überstehen. Bei den größeren weisen jeweils zwei sich gegenüberstehende Paare geringe Größenunterschiede auf. Verbindet man im Querschnitt die Mittelpunkte der zum Röhreninneren gerichteten Oberflächen, so erhält man kein Quadrat, sondern einen Rhombus. Da dieser Sachverhalt bei allen untersuchten Arten und bei sämtlichen Zahnkränzen desselben Individuums gleichbleibend ist, dürfte er kaum zufälliger Natur sein. Er erinnert vielmehr daran, daß die pyramidenförmigen Conulata ja teilweise einen rhombischen Querschnitt hatten.

Der direkte Vergleich mit den Conulata ist in den schematischen Zeichnungen der Abbildung 7a, b durchgeführt. Der Querschnitt durch einen Zahnkranz von Stephanoscyphus (Abb. 7b) zeigt einmal die genaueren Details des strukturellen Aufbaues der Peridermröhre aus den beiden erwähnten Hauptschichten. Die Zahnbildungen bestehen ausschließlich aus der inneren Schicht; sie sind hohl, das heißt mit Flüssigkeit ausgefüllt. Der Weichkörper ist angedeutet, und man erkennt aus der Lage der vier dreieckigen Septalmuskeln, daß die kleinen Zähne in den Interradien liegen, die großen in den Perradien. Im Querschnitt durch ein Conulariidengehäuse (Abb. 7a) sind die Mündungsklappen eingezeichnet, um zu verdeutlichen, daß die Mittellinien in den Interradien liegen. Diese Elemente sind ja bekanntlich als Muskelmarken zu betrachten, in denen die Klappenschließmuskel am Gehäuse angesetzt haben. Dementsprechend sind bei manchen Arten auf der Innenseite des Gehäuses Septalleisten ausgebildet. Die Kanten des Gehäuses, die meistens als Furchen mit Peridermverstärkungen ausgebildet waren, liegen in den Perradien. Der in der Zeichnung durchgeführte Vergleich zeigt eindeutig, daß im Querschnitt die großen Zähne von Stephanoscyphus den Kanten, die kleinen Zähne den Mittellinien des Gehäuses der Conularien homolog sind. Es kann kaum einem Zweifel unterliegen, daß diese Homologie ein Anzeichen dafür ist, daß Stephanoscyphus auf vierseitige Conulata zurückgeht, auch wenn in seiner Röhre die Längselemente von Kanten und Mittellinien nicht vollständig, sondern nur in Form der vertikal aufgegliederten Zahnkränze erhalten geblieben sind.

Von Interesse ist dabei, daß die Zahnkränze nur im Basalteil der Röhre auftreten. Wenn man auch annehmen und bejahen kann, daß sie eine Stützfunktion haben und das Zusammenquetschen der Röhre bei einem mechanischen Angriff verhindern, so bleibt damit noch immer ungeklärt, warum sie nur im Basalteil der Röhre gebildet werden. Es liegt daher nahe, die Zahnkränze als historisch bedingte Rudimente von Gehäuseelementen der Conulata zu betrachten, die der rezente Polyp nur noch in seiner Jugendphase erzeugt. In jedem Fall hat sich in den Zahnbildungen der im Querschnitt runden Röhre von Stephanoscyphus noch ein Rest der tetrameren (bzw. unter Einbeziehung der Mittellinien oktomeren) Symmetrie des Conulatengehäuses erhalten.

Die Zeichnungen der Abbildung 8 a $-c$ können zunächst nochmals die Einzelheiten der Oberflächenstruktur mit ihrer typischen Quer- und Längsstreifung erläutern. Sie ist im Prinzip bei allen untersuchten Arten gleich, auch wenn in der Breite der Querringe, in der mehr oder weniger scharfen Ausprägung des Quer- und Längsmusters deutliche Artunterschiede erkennbar sind (s. p. 326). Die Skizzen sollen aber noch mehr veranschaulichen, weil sie die Ergebnisse von einfachen Experimenten wiedergeben.

(a) Bei der Beschreibung der Conulatengehäuse wird immer wieder hervorgehoben (vgl. z. B. KiderLeN, p. 127), daß bei vielen Exemplaren der untere Teil durch sekun- 
där gebildete Querschotts abgeschlossen ist. Als ursächliche Erklärung wird übereinstimmend angegeben, daß die herangewachsenen Tiere vom festsitzenden Basalteil abgebrochen sein müssen, und daß die so entstandene untere Offnung des Gehäuses durch die Neubildung einer Peridermquerwand abgedichtet wurde. Die gleiche Fähigkeit können wir beim lebenden Stepbanoscyphus direkt beobachten. Trennt man einen

a
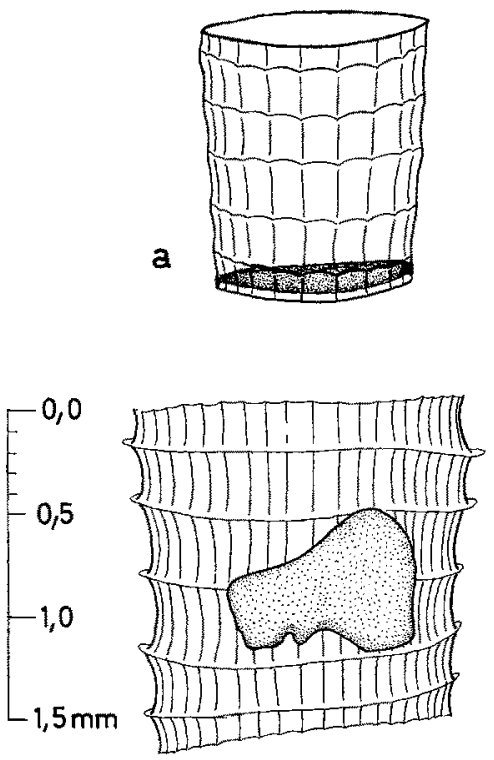

b

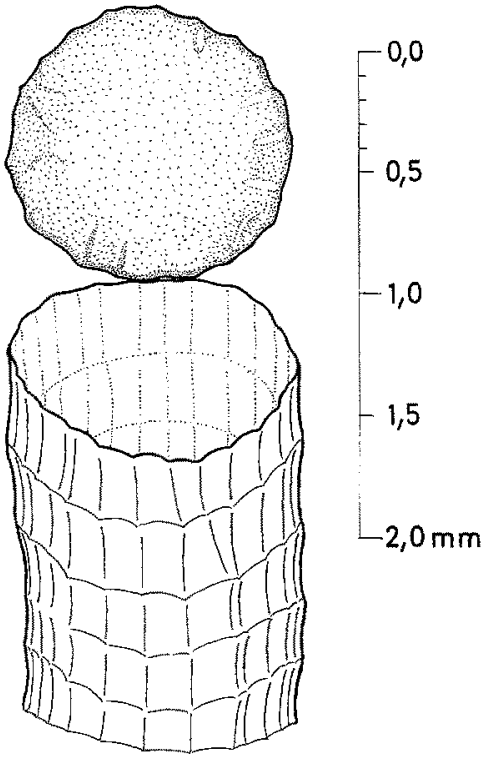

c

Abb. 8 a-c: Stephanoscyphus, experimentelle Beeinflussung der Peridermbildungen und die Deckelbildung. a oberer Röhrenteil des Polypen yon Nausitboë punctata nach Querdurchtrennung, dessen untere Offnung durch ein sekundär gebildetes Querschott (punktiert) verschlossen wurde, schematisch. $\mathrm{b}$ Röhrenstück des Polypen von Atorella vanhoeffeni mit Verschluß eines experimentell gesetzten Defektes durch eine strukturlose Peridermhaut. c Deckelbildung des Polypen von Nausithoë punctata. Einzelheiten im Text

Polypen experimentell durch (Abb. 8a, vgl. Abb. 11), so wird die künstlich hergestellte untere Offnung der oberen Polypenhällte nach wenigen Tagen durch eine Querwand aus neugebildeter Peridermsubstanz wieder verschlossen. In der gleichen Weise ist vermutlich auch die Entstehung von mehreren Querschotts zu erklären, die dem unteren Teil von manchen Conulata eine gekammerte Beschaffenheit verleihen. Es ist durchaus denkbar, daß sich der Weichkörper sekundär und zufällig von dem zuerst gebildeten unteren Querschott abgelöst und sich etwas kontrahiert hat, so daß eine weitere, höher gelegene Querwand nötig wurde, um ihm unten den notwendigen Halt zu geben. Dieser Prozeß kann mehrmals nacheinander stattgefunden haben, womit die Entstehung einer Kammerung ihre zwanglose Erklärung findet. Dieser Deutungsversuch setzt allerdings voraus, daß die beschriebene Kammerung kein ausgesprochenes Artmerkmal darstellt. 
(b) Von den Conulata wird ferner beschrieben, daß seitliche Defekte des Gehäuses durch die Bildung einer glatten, strukturlosen Peridermhaut verschlossen werden konnten (vgl. Kiderlen, p. 124). Das gleiche läßt sich am lebenden Stephanoscypbus experimentell reproduzieren (Abb. 8b). Entfernt man mit feinen Pinzetten ein Stück der Peridermröhre, so wird der Defekt nach wenigen Tagen durch die Absonderung eines Peridermhäutchens beseitigt. Daß dem neugebildeten Peridermstück außen die Oberflächenstruktur aus Quer- und Längsstreifung fehlt, ist dadurch bedingt, daß das Ersatzstück nur von den im Ektoderm der Körperwand liegenden Drüsenzellen erzeugt wird, die die glatte strukturlose Innenschicht der Peridermwandung liefern (p. 326).

(c) Wenn schließlich gesagt werden mußte, daß Stephanoscyphus sich durch das Fehlen von Deckelklappen grundsätzlich von den Conulata unterscheidet, so zeigt Abbildung $8 \mathrm{c}$, daß der Polyp die Fähigkeit, Peridermdeckel zu bilden, nicht vollständig verloren hat. Es wurde beobachtet, daß sich der Polyp zuweilen ohne erkennbare äußere Ursache in die Röhre zurückzieht und dann über Monate hinweg in einem inaktiven Zustand verharrt. In einem solchen Fall wird die Mündung durch einen runden Deckel verschlossen, wie die Zeichnung des oberen Röhrenteils mit dem abpräparierten Deckel erkennen läßt. Ein direkter Vergleich mit den dreieckigen Mündungsklappen der Conulata ist freilich nicht möglich; doch zeigt die Beobachtung, daß die Fähigkeit der Deckelbildung in abgeänderter Form erhalten geblieben ist. Uberdies wird die Röhrenmündung während des Strobilationsvorganges regelmäßig durch einen Deckelapparat verschlossen, worauf später noch kurz einzugehen ist (s. p. 334).

Abschließend kann gesagt werden, daß die dargestellten Einzelheiten über Form, Aufbau und Bildungsweise der Peridermröhre von Stephanoscyphus zusammen mit den Resultaten der Durchschneidungs- und Defektversuche eine weitgehende Ubereinstimmung mit den 'Tatsachen ergeben haben, die aus allen Beschreibungen der Gehäuse der Conulaten bekannt sind. Schon daraus darf auf seine direkte Herkunft von dieser fossilen Gruppe geschlossen werden. Wollte man daher an dem Resultat von KIDERLEN (p. 155) festhalten: „Aus den Conularien, den Paläoscyphozoa, entstanden durch Reduktion des Außenskeletts die Neoscyphozoa" (im Original gesperrt), so müßte Stephanoscyphus auf Grund dieses Kriteriums als lebendes Palaeoscyphozoon bezeichnet werden.

\section{Der Weichkörper}

Die Peridermröhre ist aber nur ein Außenprodukt des Weichkörpers. Stellt sie selbst ein altertümliches Merkmal dar und enthält sie Eigenschaften, die die Herkunt des Erzeugerorganismus von den fossilen Vorfahren erschließen lassen, so muß dieser in seiner Organisation selbst den historischen Zusammenhang noch deutlicher oder zumindest ebenso deutlich widerspiegeln. Das trifl für Stephanoscyphus in der Tat zu, auch wenn wir uns in dieser Hinsicht mit einem Indizienbeweis begnügen müssen.

Der Weichkörper (Abb. 9 a-d) ist sehr einfach gebaut und besteht aus einem dünnwandigen, sehr schlanken Sack, der mit seiner Außenwand der Peridermröhre dicht anliegt, ohne aber mit dieser fest verwachsen zu sein. Eine feste Verbindung zwischen Weichkörper und Röhre besteht nur im Basalteil. An der Mündung ist der Kopfteil durch ein annähernd ebenes Mundfeld verschlossen, das den zentral gelegenen Mund 
trägt. Daß ein Mundkegel fehlt und der Mund lediglich eine sich durch ringblendenähnliche Tätigkeit der Muskulatur erweiternde und schließende Offnung darstellt, ist zweifellos ein primitives Merkmal. Denn eine ähnliche Beschaffenheit hat sehr wahrscheinlich auch das Mundfeld der Conulata gehabt, da ein ausgesprochener Mundkegel, wie ihn der rezente Scyphistoma besitzt, schwerlich mit dem Vorhandensein der dreieckigen Mündungsklappen und ihrem Verschlußmechanismus vereinbar ist.

Das Mundfeld ist von einem Kranz aus zahlreichen Tentakeln umgeben, die bei einer Kontraktion des Polypen vollständig in den Gastralraum eingeschlagen werden

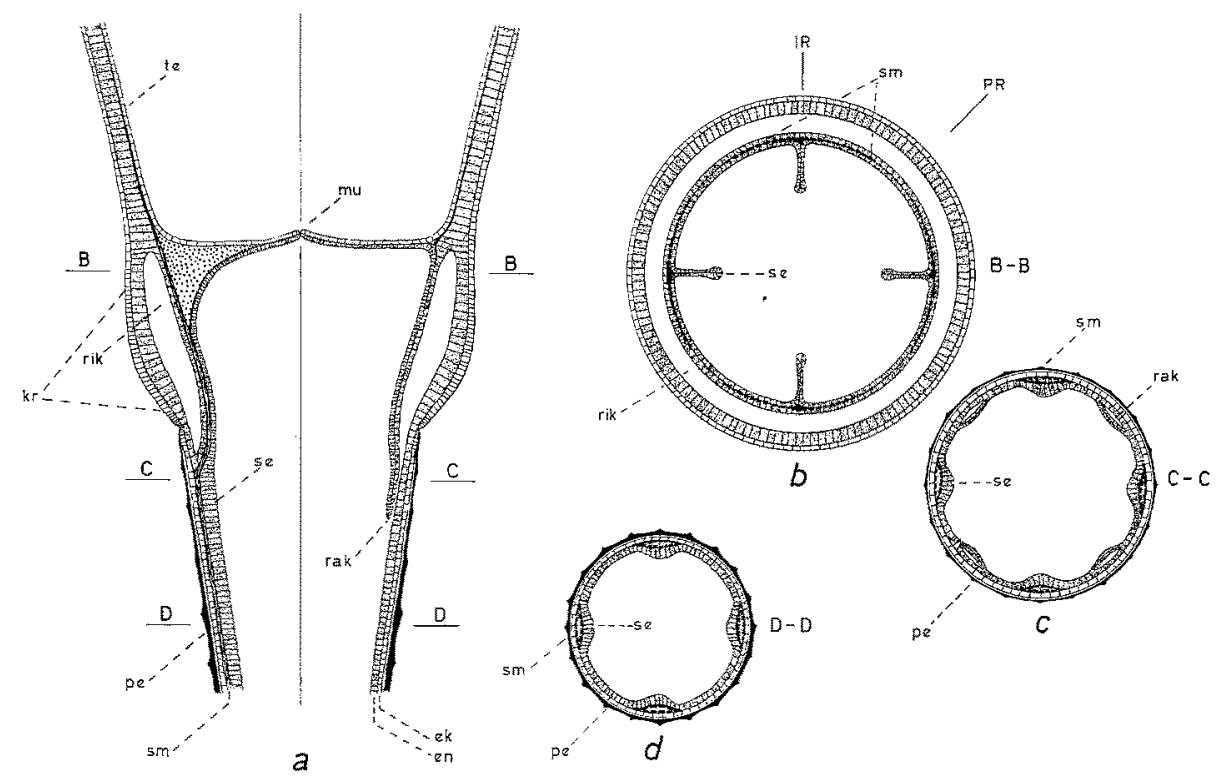

Abb. 9 a-d: Organisation des Weichkörpers von Stephanoscyphus, schematisch; gezeichnet nach Längs- und Querschnitten durch den Kopfteil des Polypen von Nausithoë punctata. a Längsschnitt; die linke Hälfte ist durch einen Interradius, die rechte durch einen Perradius gelegt. $b$ Querschnitt durch die Ebene B-B der Abbildung a; $c$ Querschnitt durch die Ebene $\mathrm{C}-\mathrm{C}$ der Abbildung a; $d$ Querschnitt durch die Ebene D-D der Abbildung a. IR Interradius, PR Perradius, ek Ektoderm, en Entoderm, kr Kragen, mu Mundöffnung, pe Peridermröhre, rak Radialkanal, rik Ringkanal, se Entodermseptum, sm Septalmuskel, te Tentakel

können, wobei sich der Mund bis an den äußeren Rand des Mundfeldes erweitert (vgl. Allman 1874, Fig. 5, Taf. 14). Diese Reaktion des vollständigen Einschlagens sämtlicher Tentakel in den Gastralraum, die regelmäßig bei der Nahrungsaufnahme oder bei einer äußeren Reizung beobachtet werden kann, ist einmalig und meines Wissens unter allen rezenten Cnidariern auf Stephanoscyphus beschränkt. Auch dieses Verhalten kann als altertümlich gedeutet werden, wenn man bedenkt, daß bei den Conulata während des Verschlusses der Mündung durch den Klappenapparat sehr wahrscheinlich der ganze Rand des Kopfteils mit sämtlichen Tentakeln nach innen eingeschlagen und umgestülpt war.

Wesentlich ist weiterhin, daß der Weichkörper mit den vier Gastralsepten und 
den in ihrer Basis gelegenen vier Septalmuskeln, die sich in ganzer Länge des Körpers vom Mund bis in den Basalteil erstrecken, eine ausgesprochen tetrasymmetrische Organisation aufweist. Die Gastralsepten ragen allerdings im größten Teil des Körpers entsprechend seinem zarten Bau nur wenig ins Lumen des Gastralraumes vor. Nur im Kopfteil springen sie von der Wand des Magens zum Mund hin bogenförmig vor, so daß sie hier den Magenraum in vier Gastraltaschen aufteilen. Das Vorhandensein von Entodermsepten und Septalmuskeln ist also keineswegs an die Existenz von Skelettelementen gebunden, wie sie bei manchen Conulata in der Form der Septalleisten der Mittellinien ausgebildet sind. Die Schlußfolgerung von KIDERLEN (p. 148), daß dem Weichkörper der Conulata Entodermsepten und Septalmuskeln gefehlt haben, wenn keine Mittellinien, Septalleisten und Mündungsklappen des Gehäuses erkennbar sind, erscheint daher nicht unbedingt zulässig. Was überdies für den Scyphistoma gilt (p. 321), trifft in gleicher Weise für Stephanoscyphus zu: Existenz und Vierzahl der Gastralsepten und Septalmuskeln sowie die ektodermale Natur der letzteren sind am besten auf der Basis des historischen Zusammenhanges mit den tetrasymmetrisch gebauten Vorfahren verständlich.

Septaltrichter, wie sie in typischer Form beim Scyphistoma ausgebildet sind, fehlen bei Stephanoscyphus. Auch darin dürtte sich diese Form den Conulata unmittelbar anschließen; denn da die Muskelstränge für den Klappenverschluß bei ihnen aller Wahrscheinlichkeit nach direkt an den Mündungsklappen einerseits, an den Mittellinien beziehungsweise an den Septalleisten andererseits angesetzt haben, erscheint die Existenz von Septaltrichtern überflüssig und wenig plausibel.

Ein besonders eigenartiges Merkmal, das unter allen Cnidarierpolypen auf Stephanoscypbus beschränkt bleibt, ist die Ausbildung eines wohlentwickelten Kanalsystems aus einem Ringkanal und vier Radialkanälen im Kopfteil. Der Ringkanal ist vom übrigen Gastralraum vollständig getrennt und mündet in ihn lediglich durch vier perradial gelegene kleine Ơffnungen der Radialkanäle ein, wodurch die radialen Symmetrie-Ebenen eindeutig bestimmt sind.

Ein solches Kanalsystem gibt es bei keinem anderen Cnidarierpolypen, wohl aber bei allen ihren Medusen; das heißt wir haben in Stephanoscyphus einen P ol y p e n $\mathrm{mit} \mathrm{dem} \mathrm{K}$ an a ls y s e m einer Meduse vor uns. Das beweist einmal die morphologische Gleichwertigkeit von Polypund Meduse; eine Meduse ist nichts anderes als ein zum Zweck der Verbreitung und Fortpflanzung abgelöster und in Anpassung an die pelagische Lebensweise umgewandelter Polyp. Was bereits von den Klassikern der Morphologie und Entwicklungsgeschichte gegen Ende des letzten Jahrhunderts, besonders von WeIsmans und den Brüdern Hertwig, auf Grund der Homologie der Organsysteme theoretisch erschlossen wurde, findet damit bei Stephanoscyphus die unmittelbare und überzeugende Bestätigung.

Zum anderen wird mit Hilfe dieses altertümlichen Merkmals jetzt endlich klar, warum bei den anderen Scyphozoen und weiterhin auch bei den Hydrozoen aus dem Polypen mit seinem wenig gegliederten oder überhaupt ungegliederten Magenraum in der Medusenknospung plötzlich Medusen hervorgehen, deren Gastralsystem durch Ringkanal und Radialkanäle in auffälliger Weise gegliedert ist. Bei der Bildung der Meduse wird somit bei allen anderen Cnidariern eine im Erzeugerpolypen nur latent vorhandene Anlage verwirklicht, die Stephanoscyphus als einziger Polyp realiter be- 
sitzt. Hydroid- und Scyphopolypen ohne ein solches Kanalsystem müssen als sekundär vereinfacht gelten und müssen von Vorfahren abstammen, die ein ähnliches Kanalsystem wie Stephanoscyphus besessen haben.

Ob die Conulata über ein derartiges Kanalsystem im Kopfteil verfügten, läßt sich nicht feststellen, da Abdrücke des Weichkörpers, falls überhaupt vorhanden, kaum ein solches Strukturdetail hinterlassen haben dürtten. Denkbar wäre es durchaus; denn bei Stephanoscyphus hat das Kanalsystem mit großer Wahrscheinlichkeit die Funktion eines hydrodynamischen Stützsystems für den Kopfteil mit seiner großen Tentakelkrone und spielt eine entsprechende Rolle vermutlich auch beim Wiederausstrecken nach einer Kontraktion. Unter diesem Gesichtspunkt könnte ein Kanalsystem mit einer solchen Funktion auch bei den Conulata das Ausstrecken des Kopfteils bei der Öffnung des Klappenverschlusses erleichtert haben. Ob die elastische Spannung des an der Mündung sehr dünnen Periderms allein für das Wiederöffnen des bei manchen Arten recht komplizierten Faltklappenverschlusses und für das Wiederausstrecken ausgereicht hat, erscheint vom funktionellen Standpunkt keineswegs so sicher, wie es bisher allgemein angenommen wurde.

Das fast vollständige Fehlen einer Stützschicht (Mesogloea) zwischen Ekto- und Entoderm, die beim rezenten Scyphistoma recht kräftig entwickelt ist und hier als Kompensationserscheinung gedeutet werden muß (CHAPMAN 1966), verstärkt schließlich ebenfalls noch den Eindruck der altertümlichen Organisation von Stephanoscyphus.

Zur Lebensweise ist noch kurz zu erwähnen, daß Stephanoscyphus seine Nahrung, kleine Planktonorganismen, vor allem Crustaceenplankton, mit Hilfe der Nesselzellen erbeutet, die in großer Zahl im Ektoderm der Tentakel und der übrigen Außenepithelien des Kopfteils liegen. Durch Kontraktion der Tentakel und das beschriebene ruckartige Einschlagen werden die Beutetiere als ganze in den Magenraum hinein befördert, wobei sich der Mund bis zum Rand des Mundfeldes erweitert. Auf dem gleichen Wege werden die unverdaulichen Reste der Nahrung ausgeschieden.

\section{Die Strobilation}

Zum Schluß muß noch kurz auf die ungeschlechtliche Vermehrung durch Strobilation eingegangen werden, durch die sich Stephanoscyphus als echter Scyphopolyp ausweist. Der Vorgang der Strobilation beim Scyphistoma der Semaeostomeae und Rhizostomeae durch die von oben nach unten fortschreitende Querteilung des Polypenkörpers kann als bekannt vorausgesetzt werden. Jede Querscheibe wandelt sich zu einer kleinen Meduse mit Randlappen und Sinnesorganen um, die nach der Ablösung während ihres pelagischen Daseins zur vollen Größe und Geschlechtsreife heranwächst. Alle Erklärungsversuche der Tatsache, daß gerade bei den Scyphozoen und nur bei ihnen die terminale Art der Medusenbildung durch Querteilung des Polypenkörpers vorkommt und nicht etwa die laterale Medusenknospung wie bei den Hydroidpolypen, sind bisher mehr oder weniger hypothetisch und wenig befriedigend geblieben. Wenn wir dagegen die Strobila von Stephanoscypbus betrachten (Abb. 10), wird der Grund für diese Besonderheit der Scyphozoenentwicklung klar. Die Peridermröhre, die bei aller Feinheit der Wandung doch eine feste und zähe Beschaffenheit aufweist, machte 
eine laterale Knospung unmöglich. Die terminale Medusenbildung läßt sich daher evolutionistisch am besten mit dem Vorhandensein der Röhre erklären, weil der Polypenkörper nur oben durch die Röhrenmündung mit der Außenwelt in Verbindung stand, so daß Vermehrungsprodukte, seien sie ungeschlechtlicher oder geschlechtlicher

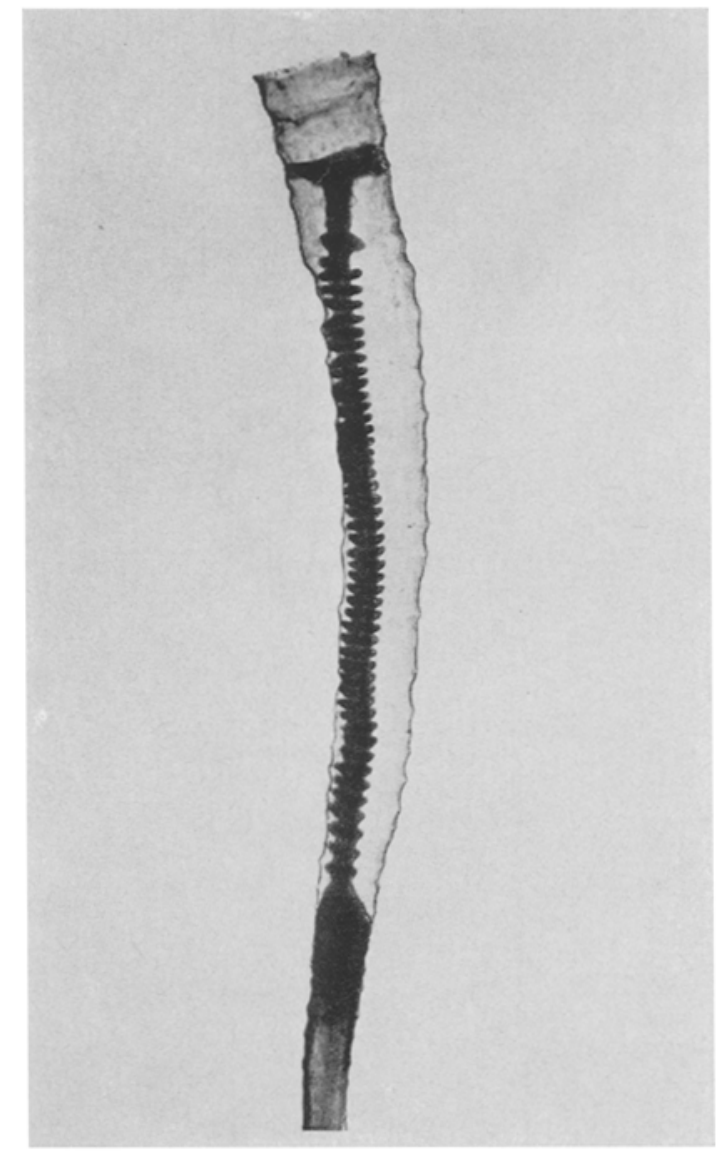

Abb. 10: Stephanoscyphus, Strobila eines jungen Polypen der Coronatenmeduse Nausithoë punctata. Das Lebendphoto gibt einen guten Eindruck von der zarten Wandung der Peridermröhre. Beachte den vom Kopfteil des Polypen gebildeten Deckelapparat, durch den die Röhrenmündung vollständig abgeschlossen ist

Natur, nur hier austreten konnten. Bei der schlanken Form des Polypen bot sich die Medusenbildung durch Querteilung des Weichkörpers mit der von oben nach unten fortschreitenden Bildung und Ablösung der Jungmedusen wohl als der gangbarste Weg an.

Die Abbildung 10 läßt deutlich erkennen, daß die polydiske Strobilation bei Stephanoscyphus prinzipiell in der vom Scyphistoma bekannten Weise abläuft, ferner aber, daß die Röhrenmündung dabei stets durch einen Deckelapparat verschlossen 
wird, der aus dem Kopfteil des Polypen entsteht. Er wird primär vom Zellgewebe des Mundfeldes und des sich darunter anschließenden Abschnittes des Kopfteils gebildet, wobei die Tentakel reduziert werden und der Mund verwächst. Vom Mundfeld wird uberdies nach außen ein feiner Peridermdeckel abgeschieden. Gegen Ende der Strobi-

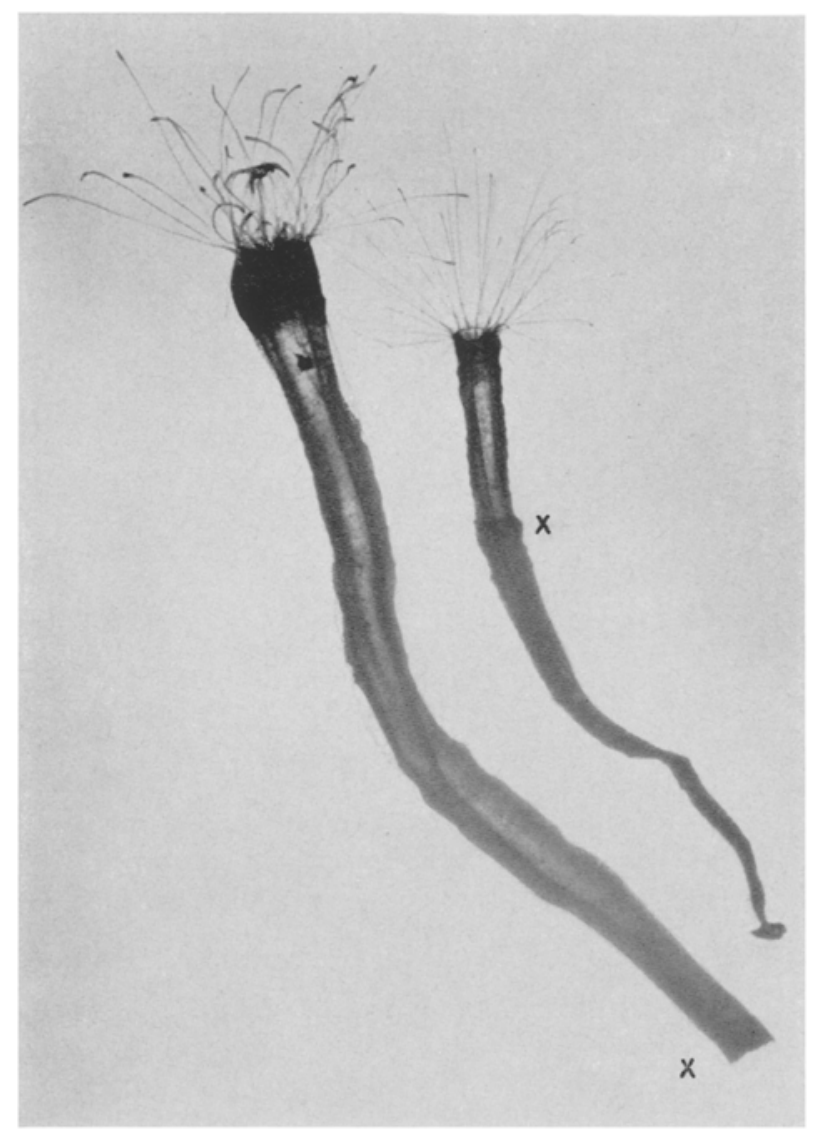

Abb. 11: Stephanoscyphus, experimenteller Nachweis der Regenerationsfähigkeit des Polypen von Nausithoë punctata. Der rechts abgebildete kleinere Polyp ist bei $\mathrm{x}$ vom größeren oberen Teil abgetrennt und hat einen neuen Röhrenabschnitt und Kopfteil regeneriert

lation verfällt der Deckelapparat einer allmählichen Degeneration und wird von den durch aktive Schwimmbewegungen austretenden Jungmedusen leicht durchbrochen.

Die Voraussetzung für die evolutionistische Entstehung dieser Art der ungeschlechtlichen Vermehrung des Polypen durch die terminale Strobilation ist offenbar in der Fähigkeit der Regeneration des Polypen bei einer Querteilung zu suchen (vgl. THIEL 1966). Sie tritt zwar beim rezenten Stephanoscypbus nicht mehr regulär in Erscheinung, wohl aber accidentell. Teilt man einen Polypen quer durch, so schließt der obere Teil der künstlich entstandene untere Offnung durch ein Querschott ab (p. 329) 
und vermag in der Kultur am Boden liegend ohne jede Beeinträchtigung seiner Lebensfunktionen weiterzuexistieren. Der untere Teil aber regeneriert nach wenigen Tagen einen neuen Kopfteil mit Tentakelkrone, Mund und Kanalsystem. Das Ergebnis eines solchen in physiologischer wie theoretischer Hinsicht wichtigen Regenerationsversuches ist in Abbildung 11 dargestellt.

Es muß noch erwähnt werden, daß es gelang, die von den Polypen durch Strobilation erzeugten Jungmedusen bis zur Geschlechtsreife aufzuziehen, auf diese Weise erstmals Arten von Stephanoscyphus bekannten Medusenspezies zuzuordnen und damit die Systematik der Gattung Stephanoscyphus auf eine gesicherte Grundlage zu stellen. Bisher wußte man lediglich, daß Stephanoscyphus die Polypengeneration der Medusenordnung Coronatae darstellt (Einzelheiten bei Werner 1967).

Wie die vorangegangene Beschreibung gezeigt hat, konnte ein großer Teil des Lebenszyklus von Stephanoscyphus im Kulturexperiment verfolgt werden. Es war sogar möglich, die Medusen zur Abgabe reifer Geschlechtsprodukte zu bringen. Die befruchteten Eier entwickelten sich zu freischwimmenden Planulalarven weiter, die jedoch abstarben, ehe sie zur Anheftung und zur Umwandlung in den jungen Polypen kamen. Wie seit langem bekannt (Metschnikoff 1886), erfolgt die Weiterentwicklung des planktischen Larvalstadiums von Stephanoscyphus in der für alle Cnidarier üblichen Weise, daß sich die Planula mit dem bei der Schwimmbewegung vorangerichteten aboralen Pol anheftet, und daß am gegenüberliegenden oberen Pol der Mund und die Tentakel gebildet werden. Die Entwicklung konnte ron Metschnikoff bis zu einem frühen Stadium weiterverfolgt werden, auf dem der junge Polyp eine kleine basale Haftscheibe und ein feines Peridermrohr besaß, in das er sich zurückziehen konnte.

\section{DISKUSSION UND ERGEBNIS}

Wenn man bisher angenommen hat, daß der bekannte Scyphistoma weitgehend den ursprünglichen Typus des Scyphopolypen repräsentiere (CHApman 1966, THex 1966), so kann es nunmehr keinen Zweifel daran geben, daß Stephanoscyphus sowohl durch den Besitz seiner Peridermröhre wie auch durch die Organisation seines Weichkörpers und durch seine Entwicklungsgeschichte den Vorfahren der rezenten Scyphozoen am nächsten steht. Wie seit langem bekannt, haben auch die Coronatenmedusen eine altertümliche Form bewahrt, was etwa in dem von HAECKEL geprägten Genusnamen Palephyra zum Ausdruck kommt. Daraus muß geschlossen werden, daß die Ordnung Coronatae den ältesten und ursprünglichsten Seitenzweig der evolutiven Entwicklungslinie darstellt, die von den Conulata zu den rezenten Scyphozoen hinführt. Insbesondere dürfte nach allem kaum ein Zweifel daran möglich sein, daß Stephanoscyphus als direkter Nachkomme dieser fossilen Vorfahren betrachtet werden muß.

Umgekehrt spricht die Existenz dieses rezenten Polypen, der so überraschend viele Ahnlichkeiten mit den Conulata besitzt, unbedingt für deren Scyphozoennatur. Wäre Stephanoscyphus schon früher besser bekannt und untersudht gewesen, so hätte das die richtige systematische Einordnung der Conulata sidher zu einem fruheren Zeit- 
punkt und mit besseren Argumenten ermöglicht, als sie KIDERLEN (1937) zur Verfügung standen.

Wie bereits einleitend kurz erwähnt, haben neuerdings TERMIER \& TERMIER (1948) die Scyphozoennatur der Conulata erneut bezweifelt und sind unter eingehender Erörterung aller Gesichtspunkte zu dem Resultat gelangt, daß die Conulata am ehesten mit den rezenten Pterobranchia (Hemichordata) zu vergleichen seien.

Mit der Scyphozoennatur der Conulata sind nach Ansicht dieser Autoren folgende Tatsachen unvereinbar.

(a) Es sind keine rezenten Cnidaria bekannt, deren Skelett Phosphat enthält.

Einwand: Nach den bisherigen Kenntnissen besteht das Gehäuse von Stephanoscyphus in der Tat nur aus Chitin. Wie aber Chapman (1966, p. 56) erwähnt hat, trifft das Fehlen von Phosphat nicht für sämtliche rezenten Cnidaria zu. Nach den Zusammenstellungen von VINOGRADOw (1953) kommt jedenfalls Phosphat in rezenten Korallen in geringen Mengen oder Spuren vor. Außerdem muß mit der Möglichkeit gerechnet werden, daß bereits bei den fossilen Arten, die als Verbindungsglieder zwischen den vierseitigen Conulata und Stephanoscyphus existiert haben müssen, kein Phosphat mehr in die Gehäuse eingelagert wurde.

(b) Bei keinem Cnidarier kommt das Größenwachstum der Gehäuse durch "segments alternes" vor, das für die Conulata typisch ist. Was darunter zu verstehen ist, haben die Autoren (pp. 720-721) ausführlich erläutert. Es ist das Längenwachstum durch periodische, diskontinuierliche Ausscheidung der Gehäusesubstanz aus Drüsenzellen, dessen Ausdruck die Querstreifen der Oberflächenstruktur sind.

Gegenargument: Genau die gleiche Art der Oberflächenstruktur aber treffen wir bei Stephanoscyphus an (p. 325). Das Längenwachstum durch successive Bildung der Querstreifen konnte verifiziert und durch die direkte Beobachtung ursächlich erklärt werden. Ebenso war es möglich, die Längsstreifung der Oberfläche, die von vielen Conulata bekannt ist, bei Stephanoscyphus in ihrer Entstehung zu deuten. Es spricht für sich, daß die Bildungsweise der Oberflächenstruktur der Peridermröhre von Stephanoscyphus realiter genau von der Art ist, wie sie die Autoren für die Conulata postulieren.

(c) Es gibt bei den Cnidaria kein Beispiel dafür, daß bei der Knospung die Mündung der Theca des Erzeugerpolypen eine entgegengesetzte Richtung hat wie die Mündungen der Thecen der von ihm erzeugten Sekundärpolypen. Mit diesem Sachverhalt, der der näheren Erläuterung bedarf, beziehen sich die Autoren, wenn ich sie recht verstehe, auf ihre Befunde an Conulatenmaterial aus Marokko. Bei zwei Arten, Mesoconularia arcuata und Conularia quadrisulcata, besteht nach ihrer Ansicht der Basalteil des Gehäuses aus zwei Kammern, deren Trennwand nicht quer, sondern schräg verläuf, so daß in Querschnitt tatsächlich der Eindruck des Vorhandenseins von zwei getrennten Kammern entsteht, von denen die eine nach oben, die andere nach unten geöffnet sein soll (Abb. 12). Dieser Befund wird in der Weise gedeutet, daß die eine Kammer von einem larvalen Jugendstadium, die andere aber von dem durch Knospung aus dem Jugendstadium hervorgehenden definitiven Organismus gebildet sein soll. Die Autoren werden in dieser Annahme dadurch bestärkt, daß ähnliche Bilder aus der Literatur bekannt sind. 
Gegenargument: Der der Kritik offenstehende Ansatzpunkt dieser Deutung liegt in der Annahme, daß es sich bei diesen Beobachtungen um reguläre, in der Entwicklung der Arten regelmäßig auftretende Erscheinungen handelt. Termer \& Termter haben aber nicht berücksichtigt, daß ihre Befunde auch Zufallsprodukte sein können, die zum Teil ursächlich mit dem Abbrechen der Tiere von der Basis und mit der
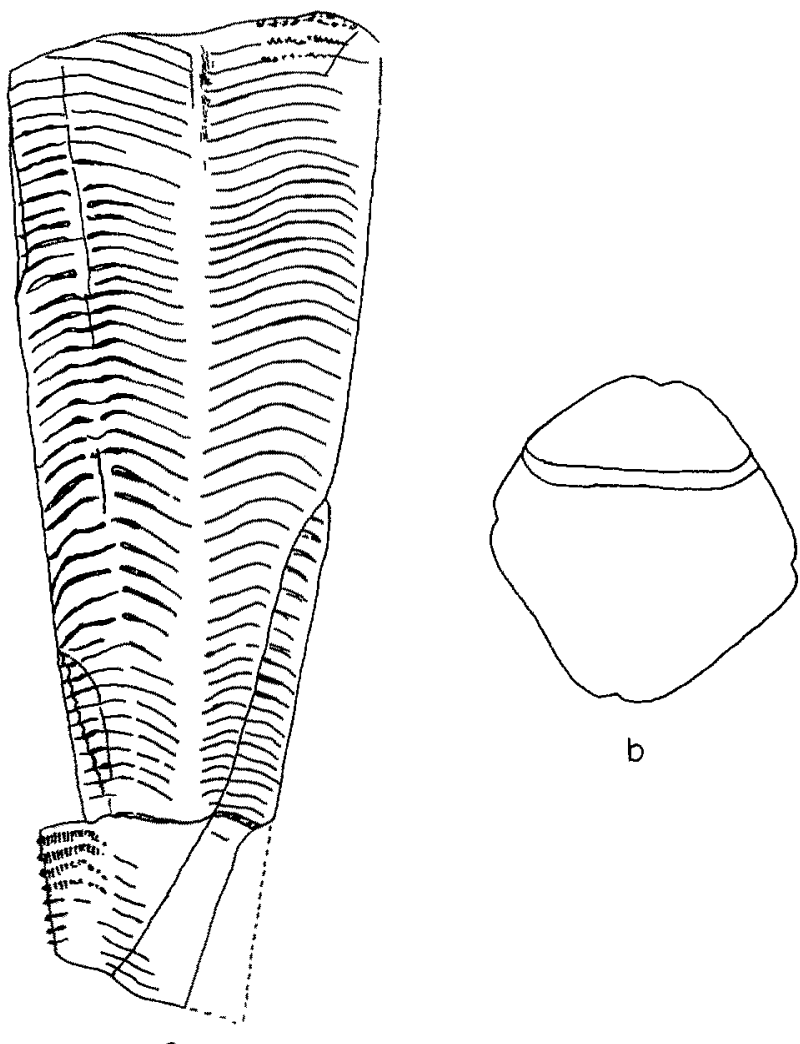

b

Abb. 12: Conularia quadrisulcata. a Seitenansicht, b Querschnitt durch den Apikalteil. (Aus Termier \& Termier 1948, p. 713, Fig. 12, 13)

Fähigkeit des basalen Teiles zusammenhängen, einen neuen Kopfteil zu regenerieren. Damit ist dann auch notwendigerweise ein neues Wachstum des Gehäuses verbunden, das aber anfangs unregelmäßiger Natur sein kann. Diese Gegenauffassung stïtzt sich auf reale Beobachtungsbefunde bei Stephanoscyphus. Wie Abbildung 5 (p. 325) deutlich erkennen läßt, hat in etwa der Mitte des dargestellten Tieres eine Unterbrechung des normalen Röhrenwachstums stattgefunden, die ausging und bedingt war von einer Kontraktion des Polypenweichkörpers in die Röhre hinein. Aus irgendeinem, nachträglich nicht mehr erkennbaren Grunde war der Polyp nicht in der Lage, sich beim weiteren Wachstum bis zur Mündung auszustrecken, wie das normalerweise der Fall 
ist. Die Ausscheidung der neuen Röhrensubstanz erfolgte entsprechend nicht am oberen Rand der Röhre, sondern in ihrem Inneren auf einem tiefergelegenen Niveau; überdies hatte der neu entstehende Röhrenteil einen geringeren Durchmesser als der alte. So erklärt sich das im Photo festgehaltene und in der Abbildung 13 nochmals erläuterte Bild. Der in der neuen Wachstumsphase erzeugte Röhrenabschnitt steckt also mit dem

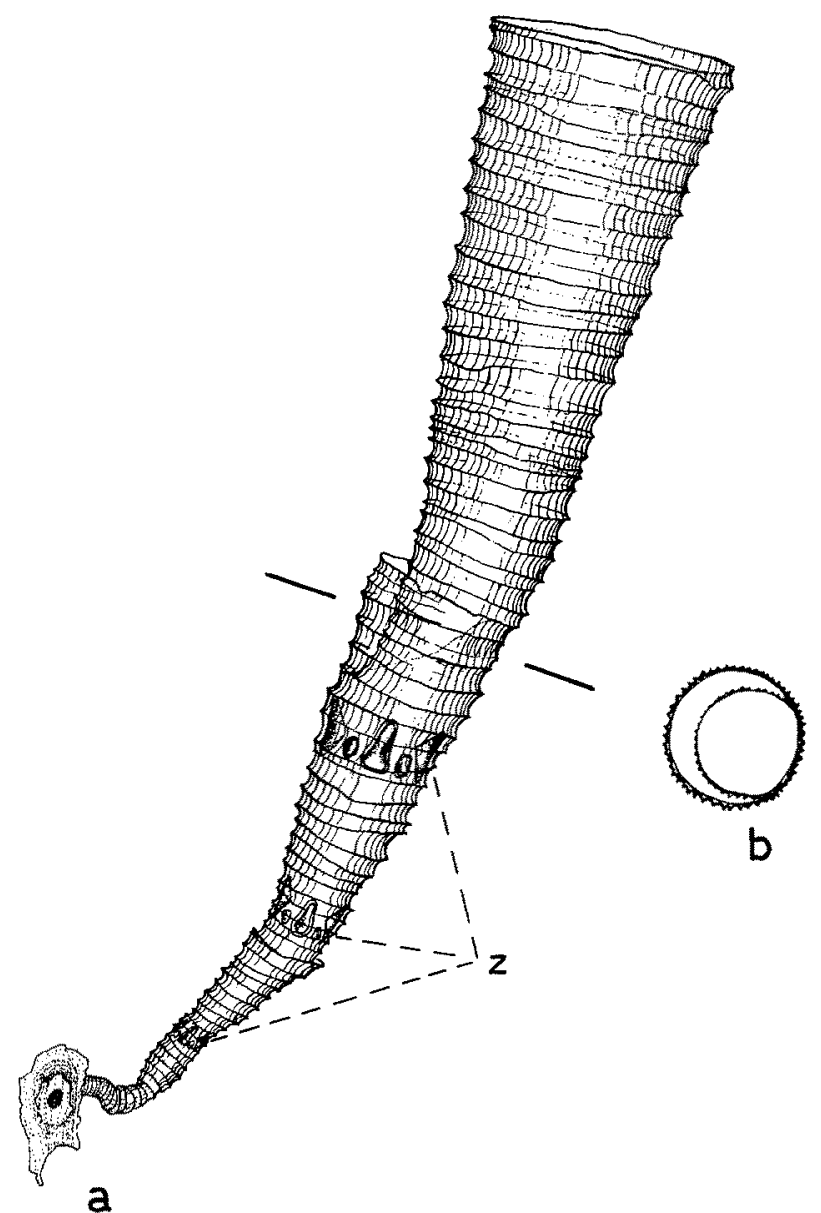

Abb. 13 a, b: Stephanoscypbus, irreguläres Wadhstum der Peridermröhre des Polypen von Atorella vanhoeffeni (vgl. das Photo der Abb. 5). a Seitenansicht, b Querschnitt, z Zahnkränze. (Gez. U. Schreiber)

unteren engeren Ende in der alten Röhre, die in der vorangegangenen Wachstumsphase bereits einen weiteren Durchmesser angenommen hatte. Die Ursache dieser vom Normalen abweichenden Gehäusebildung liegt damit in einer zufälligen Wachstumsunterbrechung des Weichkörpers eines und desselben Polypen. Legt man einen Querschnitt durch die fragliche Zone des Gehäuses, so erhält man das in Abbildung 13b dargestellte Bild, das mit dem von Termier \& Termier gegebenen Querschnitt ihrer 
Conularien (vgl. Abb. 12b) prinzipiell übereinstimmt. Übrigens zeigt die Seitenansicht des Exemplars der Abbildung 12a auf der linken Seite interessanterweise durch die verschiedene Breite des oberen und unteren Teiles des einheitlichen Gehäuses die gleiche Diskontinuität der Gehäusebildung an, wie wir sie bei Stephanoscyphus beob-

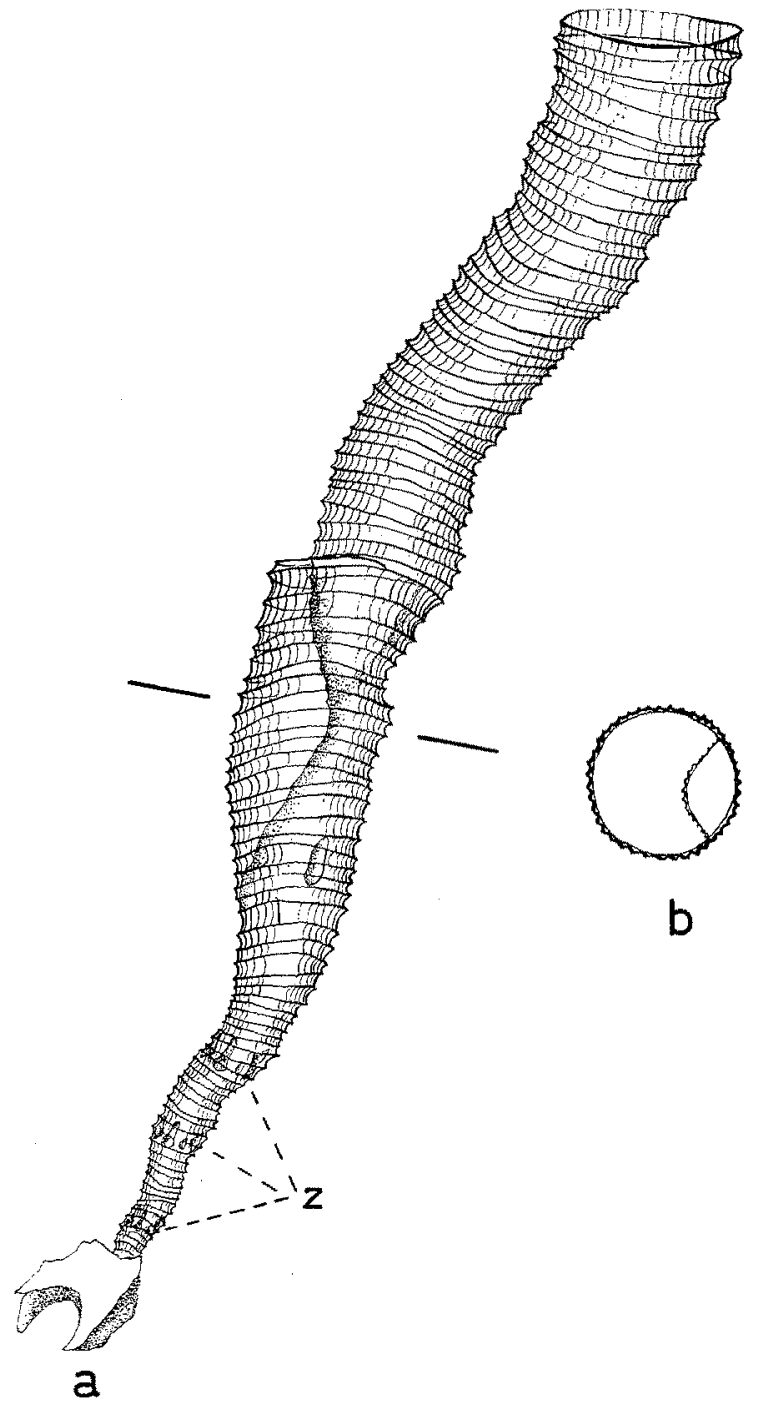

Abb. 14 a, b: Stephanoscyphus, ein extremer Fall des irregulären Wachstums der Peridermröhre des Polypen von Atorella vanboeffeni, das die Folge einer Größenreduktion des Weichkörpers ist. a Seitenansicht, b Querschnitt, z Zahnkränze. (Gez. U. SCHREiber)

achten und in der geschilderten Weise ursächlich deuten konnten. Ein noch extremeres Beispiel einer solchen Diskontinuität der Röhrenbildung bei Stephanoscypbus ist in Abbildung 14 wiedergegeben. 
Auf Grund der Existenz und der Organisation von Stephanoscyphus muß daher auch der Annahme dieser Autoren widersprochen werden, daß die Übereinstimmung der tetrameren Symmetrie bei den Conulata und den rezenten Scyphozoen lediglich eine Konvergenzerscheinung sei. Auf der anderen Seite weisen die Gehäuse der rezenten Pterobranchia nach ihrer ganzen Form derartige Verschiedenheiten auf, daß der von Termier \& Termier vorgeschlagenen Annäherung der Conulata an diese Gruppe, damit an die Graptolithen, keine reale Grundlage zuerkannt werden kann.

Schließlich gestattet die genaue Kenntnis von Stephanoscyphus, daß wir uns von Bau und Lebensweise der Conulata ein wesentlich besseres und anschaulicheres Bild machen können, als das bisher möglich war und wohl auch für möglich gehalten wurde. Wenn wir uns einen Organismus vorstellen, dessen Weichkörper wie der von Stephanoscyphus gebaut, dessen Gehäuse pyramidenförmig war und dessen Mündung mit vier dreieckigen Peridermklappen verschlossen werden konnte, so haben wir in summarischer Annäherung einen zutreffenden Eindruck davon, wie die fossilen Conulata lebend ausgesehen haben.

KIDERLEN hat die Auffassung ausgesprochen, daß die Conulata nur eine geringe Anzahl von Tentakeln besaßen und hat dies auch in seinen Rekonstruktionsbildern zum Ausdruck gebracht (p. 321, Abb. 2). Dieser Annahme vermag ich mich nicht anzuschließen; denn einmal spricht dagegen der Befund bei Stephanoscyphus, bei dem die Tentakelzahl je nach Art 40 bis über 100 beträgt. Evolutionistisch muß dieses Merkmal zwar als abgeleitet betrachtet werden; seine Entstehung dürfte mit der im Laufe der Evolution auftretenden allgemeinen Größenzunahme der Formen zusammenhängen. Aus diesem Grunde ist anzunehmen, daß zumindest die größeren Arten der Conulata, die zum Teil ja auch eine recht weite Mündung hatten (vgl. RichTER \& Richter 1930), über eine entsprechend große Anzahl von Tentakeln verfügten. Tatsächlich hat Conchopeltis alternata, die einzige Form, bei der Abdrücke erkennbar sind, zahlreiche Tentakel besessen (vgl. MOORE \& HARrington 1956, p. F 32, Fig. 22).

Der rekonstruierbare Teil der Entwicklungsgeschichte der Conulata verlief aller Wahrscheinlichkeit nach ähnlich, wie es oben für Stepbanoscyphus beschrieben wurde (vgl. Termer \& Termier 1948, p. 718). Ein frei bewegliches Jugendstadium heftete sich an einem festen Gegenstand an, etwa an dem Gehäuse eines erwachsenen Artgenossen, und zwar wahrscheinlich mit dem in der Bewegung nach vorn gerichteten aboralen Pol. Dieser trug Drüsenzellen, die ein klebriges Sekret ausschieden, das der ersten Anheftung diente und sich zu der basalen Haftscheibe ausformte. Aus dem nach oben gerichteten Pol entwickelte sich der Kopfteil mit Mundfeld und Tentakelkrone, während die Sekretionstätigkeit der in der Epidermis der Körperwand und des Kopfteils gelegenen Drüsenzellen das Gehäuse und die Mündungsklappen entstehen ließ. Es muß betont werden, daß es sich bei den solitären Formen in der Gehäusebildung um einen einheitlichen Bildungs- und Wachstumsvorgang handelte, bei dem basal am Anheftungspol die Haftscheibe, an den Seitenflächen und am oberen Pol die übrigen Teile des Gehäuses entstanden. Dementsprechend wuchs der junge Polyp bei der weiteren Entwicklung des Weichkörpers und des Gehäuses zu einem erwachsenen Tier heran, dessen solitäre, primär und obligatorisch festsitzende Lebensweise erst dann unterbrochen wurde, als es unter der Einwirkung der zunehmenden Größe und seines 
Gewichtes einerseits, unter dem Einfluß von Außenfaktoren andererseits vom dünnen Basalteil abbrach.

Dieser übrigbleibende Basalteil, der nach wie vor mit der Haftscheibe angeheftet blieb, dürfte die Fähigkeit besessen haben, einen neuen Kopfteil des Weichkörpers zu regenerieren, der damit in eine neue Phase des Wachstums und der Gehäusebildung eintreten konnte (vgl. KiderLeN, p. 162). Die Vorgänge des Abbrechens und der

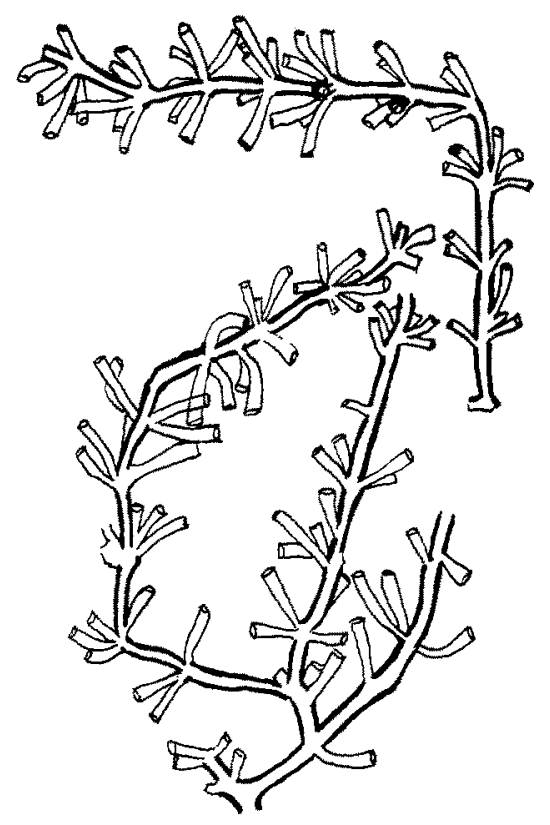

Abb. 15: Stephanoscyphis racemosus KomaI, eine koloniebildende Form.

(Aus Leloup 1937, gezeichnet nach dem Photo Fig. 41 A, p. 64)

Regeneration können sich überdies am gleichen Individuum wiederholt haben. Diese Möglichkeit, die wir für die Conulata durchaus postulieren dürfen, ändert nichts an der Grundtatsache, daß die meisten Conulata solitäre, das heißt einzellebende Organismen waren.

Das braucht nicht auszuschließen, daß es ebenso wie bei dem rezenten Stephanoscyphus (Abb. 15) auch bei den Conulata koloniebildende und verzweigte Formen gegeben hat, auch wenn die solitäre Lebensweise zweifellos die ursprünglichste war (vgl. Schmid \& Terchmüller 1958). Bei dem oft abgebildeten Fossilfund Sphenothallus (syn. Serpulites) angustifolius (p. 320, Abb. 1c) haben wir allerdings sehr wahrscheinlich keine echte Koloniebildung vor uns, wie schon von anderen Autoren bemerkt wurde. Die kleineren Exemplare sind mit Haftscheiben versehen und haben sich vermutlich als vagile Jugendstadien auf dem leeren Gehäuse eines großen Tieres der gleichen Art angeheftet, das mit dem Mündungsteil nach unten im Sediment steckte. Im Gegensatz dazu bietet die dargestellte Art Stephanoscyphus racemosus das Bild einer echten Verzweigung: An einem Hauptsproß, der sich seitlich weiter aufzweigen kann, sitzen in zahlreichen Etagen Wirtel von jeweils mehreren Polypen. 
Nach wie vor bleibt die Fortpflanzung der Conulata in Dunkel gehüllt. Wir wissen nicht, ob sich die Polypen direkt durch die Bildung von Ei- und Samenzellen geschlechtlich vermehrt haben, oder ob sie bereits in der Lage waren, durch Strobilation die getrennte Generation der Medusen zu erzeugen, auf die die geschlechtliche Vermehrung übertragen wurde. Die Tatsache, daß Coronatenmedusen erst von der Epoche des Jura $\mathrm{ab}$ fossil erhalten sind, schließt die letztere Möglichkeit nicht aus. Ein großer Teil auch der rezenten Coronatenmedusen hat nur eine geringe Größe (10 bis $30 \mathrm{~mm}$ Durchmesser). Für die von ihren Polypenvorfahren erzeugten, vermutlich ebenfalls nur kleinen Medusen dürften wohl nur geringe Chancen bestanden haben, als fossile Abdrücke bis in die Jetztzeit zu gelangen. In jedem Fall zeigt die Existenz von Stephanoscyphus, daß die evolutive Entstehung einer getrennten Medusengeneration nicht mit einer Rückbildung des Gehäuses in. Verbindung gebracht werden kann.

Indes kann ein morphologischer Unterschied zwischen den Conularien und Stephanoscyphus vielleicht Licht auf diese Frage werfen. Die Existenz des Klappenverschlusses mit der zugehörigen Muskulatur bedeutet bei den Conularien, daß der Weichkörper mindestens im Bereich der Mündung fest mit dem Gehäuse verwachsen war. Bei Stephanoscyphus aber geht das Fehlen eines Mündungsverschlusses einher mit der Fähigkeit des Polypen, den Weichkörper bei einer Kontraktion nicht nur von der Röhrenmündung, sondern auch in einem großen Teil des gesamten Körpers von der Röhrenwandung vollständig abzulösen. Eine Verwachsung zwischen Epidermis und Innenwand besteht nur im Basalteil, wo die Septalmuskel ihr Widerlager haben. Die Ablösung des Weichkörpers von der Innenwand der Peridermröhre tritt bei der Strobilation regelmäßig in Erscheinung (p. 334, Abb. 10) und ist mit einer Kontraktion und Streckung verbunden. Die Fähigkeit zur aktiven Ablösung des Weichkörpers von der Peridermröhre ist eindeutig eine Voraussetzung für diese Art der ungeschlechtlichen Vermehrung. Daher erscheint für Stephanoscyphus die evolutionistische Schlußfolgerung zulässig, daß die Entstehung der Strobilation mit dem Erwerb der genannten Fähigkeit gekoppelt und daß der Verlust der Klappenverschlußeinrichtung die notwendige Folge war. Durch diese Uberlegungen werden wir zu der Vermutung geführt, daß die Conularien noch nicht die Fähigkeit der Medusenbildung besaßen. Wir müssen daher in ihnen geschlechtsreife Polypen erblicken.

Es läßt sich auch nicht mit Sicherheit beweisen, daß die Conulata Nesselzellen besaßen, also als echte Cnidaria angesehen werden können. Der ganze Körperbau und das Vorhandensein von Tentakeln aber machen einen Beutefang und eine Ernährungsweise wahrscheinlich, wie wir sie bei Stephanoscypbus tatsächlich antreffen. Es steht der Annahme nichts im Wege, daß die Conulata auch bereits Nesselzellen besaßen.

Die Auffassung der obligatorisch sessilen Lebensweise der Conulata, die sich auf die Kenntnis von Stephanoscyphus stïtzen kann, steht im Gegensatz zu der meist vertretenen Ansicht, daß die Conulata nur in ihrer Jugend festsaßen, daß sie sich aber als erwachsene Tiere regelmäßig ablösten und dann eine vagile Lebensweise führten. Von dem im Rekonstruktionsbild KIDERLENs vorgestellten Wesen (p. 321, Abb. 2b), das ja in der Tat eine verblüffende Ahnlichkeit mit Stephanoscyphus hat, nimmt der Autor sogar an, daß es Meduseneigenschaften gehabt und sich aktiv freischwimmend bewegt habe (vgl. Richter \& RICHTER 1930).

Eine historisch und sachlich höchst interessante Parallele dazu ist übrigens, daß 
Stephanoscyphus von zoologischer Seite ebenfalls bereits einmal Meduseneigenschaften zugeschrieben sind. AlLMAN (1874) gelangte auf Grund der Anatomie zu der irrtümlichen Auffassung, daß er es mit einer völlig neuen Gruppe der Hydrozoa zu tun habe, und er errichtete deswegen die neue Ordnung Thecomedusae (vgl. p. 318).

Unbezweifelbar ist, daß sich die heranwachsenden Conulata von dem dünnen Basalteil sehr oft abgelöst haben. Der überwiegende Teil der Fossilfunde läßt die Haftscheibe vermissen und ist am unteren Ende abgerundet und häufig mit Querschotts versehen. Diese Befunde allein reichen aber schwerlich aus, die Annahme einer regulären, physiologischen, das heißt aktiven Natur des Ablösungsvorganges zu begründen. Wahrscheinlicher ist, daß die Tiere zu groß und zu schwer wurden, daß sie dadurch dem strömenden Wasser einen zu großen Widerstand entgegensetzten und so von der dünnen Basis abbrechen mußten. Sie können dann nur rein passiv am Boden getrieben sein. Die Rundung des unteren Endes deutet darauf hin, daß es nach dem Abbrechen durch Abschleifen abgerundet und durch stets neue Peridermbildung abgeschlossen wurde, was mit der Bildung der Querschotts einherging. Auf dem Boden treibend, müssen die Tiere daher noch eine kürzere oder längere Zeit am Leben geblieben sein, bis sie schließlich vom Sediment bedeckt wurden und abstarben. In anderen Fällen fehlt die Rundung, und das untere Ende ist scharfkantig geblieben; die Gehäuse müssen also bald nach dem Abbrechen eingebettet worden sein. Aus dieser Deutung ergibt sich auch die Erklärung für die keineswegs selbstverständliche Tatsache, daß so viele Conulata trotz der Feinheit ihrer Gehäuse fossil erhalten geblieben sind. Ergänzend kann mitgetellt werden, daß sich von über 50 lebenden Stephanoscyphus, die unter den Kulturbedingungen zum Teil eine Länge von über $50 \mathrm{~mm}$ erreichten, in nunmehr über zweijähriger Beobachtungsdauer kein einziges Exemplar vom Ansatzkörper aktiv abgelöst hat oder im unteren Teil von selbst abgebrochen ist.

Gegen die Annahme einer freischwimmenden Lebensweise der Conulata nach Art einer Meduse spricht der ganze Körperbau und vor allem das Fehlen einer entsprechenden Bewegungseinrichtung. Man könnte zwar wie KIDERLEN (pp. 158-159) an den Rückstoßmechanismus bei einem plötzlichen Klappenverschluß denken. Die Voraussetzung für die Erzeugung einer aktiven Bewegung auf der angedeuteten mechanischen Grundlage aber wäre gewesen, daß die Mündungsklappen mittels der zugeordneten Muskulatur Offnungs- und Schließbewegungen in sehr schneller Wechselfolge ausgeführt hätten. Gegen diese Möglichkeit spricht schon die bei manchen Arten recht komplizierte Einrichtung des Faltklappenverschlusses; abgesehen von der natürlichen Elastizität des Gehäusematerials fehlt überdies jeder Hinweis auf die Existenz eines Bewegungsmechanismus, der gegenüber der Tätigkeit der Klappenschließmuskel als Antagonist beim Offnen wirksam geworden wäre. In jedem Fall hat die direkte Beobachtung bei Stephanoscyphus ergeben, daß eine plötzliche Kontraktion des Kopfteils, verbunden mit dem ruckartigen Einschlagen der Tentakel in den Gastralraum bei vollständig geöffnetem Mund, keine Horizontalbewegung des vom Ansatzkörper abgelösten Polypen erzeugen kann. Außerdem ist zu berücksichtigen, daß sich das Wiederausstrecken nach einer solchen plötzlichen Kontraktion des Kopfteils in sehr langsamem Tempo vollzieht, wie das im übrigen für sämtliche rezenten Cnidaria die Regel ist. Es liegt kein Anlaß für die Annahme vor, daß sich die Conulata in dieser Hinsicht anders verhalten hätten. Daher bestehen gute Gründe für die Vermutung, daß das Größen- 
wachstum der Conulata, das in bewegtem Wasser zum Abbrechen von der dünnen Basis führen mußte, im Zusammenhang mit der Unfähigkeit zu aktiven Eigenbewegungen die Ursache für ihr Aussterben geworden ist.

Wir gelangen nach allem zum Endergebnis, daß die Conulata lediglich eine reine Polypengeneration repräsentiert haben, und daß auch ihre sekundär abgelösten Vertreter keinesfalls in evolutionistischem Sinne als Ubergangsstadien von Polypen zu Medusen betrachtet werden können. Vielmehr müssen alle Uberlegungen auf den basalen Teil gerichtet werden, der mit der Haftscheibe am Ansatzkörper angeheftet blieb und einen vollständigen Polypen regenerierte. Nur dieser Basalteil überlebte und konnte so Träger der evolutionistischen Veränderungen werden, die schließlich zum Erwerb der Fähigkeit der Medusenbildung führten und offenbar damit das Überleben dieser Gruppe bis in die heutige Zeit ermöglichten.

Vom Aussterben waren ganz allgemein wahrscheinlich die kleineren und schlankeren Formen der Conulata weniger bedroht, an die sich Stephanoscyphus anschließen dürfte. Denn wenn es seit der Epoche des Jura Coronatenmedusen gegeben hat, so müssen auch die sie erzeugenden Polypen existiert haben. Entweder sind sie wegen ihrer Kleinheit oder auch weil ihnen die Fähigkeit zur Einlagerung von Calciumphosphat verlorenging, fossil nicht erhalten, oder aber sie sind noch nicht entdeckt. Eine weitere Möglichkeit wäre darin zu suchen, daß sie in die Stillwasserzonen der Tiefsee ausgewichen sind, wo heute ja tatsächlich Vertreter von Stephanoscypbus leben. Von der Tiefsee könnte dann die Wiederbesiedlung des Schelfgebietes ausgegangen sein. Unabhängig aber von allen derartigen Überlegungen spekulativer Natur lassen Existenz, Organisation und Lebensweise von Stephanoscyphus keinen Zweifel daran $z u$, daß, abgesehen von der veränderten Form des Gehäuses und dem Fehlen der Mündungsklappen, der Organisationstypus der Conulata in unserem Polypen bis auf den heutigen Tag erhalten geblieben ist.

\section{ZUSAMMENFASSUNG}

1. Der rezente marine Scyphopolyp Stephanoscyphus kommt mit mehreren Arten auf dem Schelf der Kontinente und in größeren Meerestiefen vor. Es war erstmals möglich, mehrere Arten in Laboratoriumskultur zu nehmen und Morphologie, Entwicklung und Lebensweise am lebenden Objekt zu untersuchen.

2. Stephanoscyphus ist durch den Besitz einer aus Chitin bestehenden Peridermröhre ausgezeichnet, die von der Epidermis gebildet wird und den ganzen Körper von außen umschließt. Aus der Röhrenmündung wird nur der Kopfteil mit Mundfeld. und einem Kranz aus zahlreichen Tentakeln herausgesteckt. Der sich verjüngende Basalteil ist mit einer kleinen Hattscheibe angeheftet.

3. Der Besitz der Peridermröhre ist ein altertümliches Merkmal, durch das Stephanoscyphus sich an die fossilen Vorfahren der rezenten Scyphozoa, die Conulata, direkt anschließt. Die Einzeluntersuchungen über Form, Aufbau, Oberflächenstruktur und Bildungsweise der Peridermröhre haben zusammen mit den Resultaten von Durchschneidungs- und Defektversuchen eine weitgehende Öbereinstimmung mit den Tatsachen ergeben, die aus den Beschreibungen der Conulatengehäuse bekannt sind. 
4. Auch die Organisation des Weichkörpers weist altertümliche Merkmale auf. Solche sind: der tetrasymmetrische Bau mit dem Vorhandensein von vier Gastralsepten und vier Septalmuskeln; ferner das Fehlen des Mundkegels, der Septaltrichter und der Mesogloea. Ein besonders wichtiges Merkmal ist die Existenz eines Kanalsystems im Kopfteil, das aus einem geschlossenen Ringkanal und den vier von ihm ausgehenden Radialkanälen besteht. Ein solches Kanalsystem wird bei keinem anderen Cnidarierpolypen, wohl aber bei ihren Medusen gefunden. Stephanoscyphus ist ein Polyp mit dem Kanalsystem einer Meduse, womit die morphologische Gleichwertigkeit von Polyp und Meduse direkt erwiesen ist. Eine Meduse ist ein zum Zweck der Verbreitung abgelöster und in Anpassung an die pelagische Lebensweise umgewandelter Polyp.

5. Durch die polydiske Strobilation erweist sich Stephanoscyphus als echter Scyphopolyp. Existenz und Eigenart dieser terminalen, auf die Scyphozoa beschränkten Medusenbildung lassen sich evolutionistisch am besten aus dem Besitz der festen Peridermröhre ableiten.

6. Existenz, Organisation und Lebensweise von Stephanoscyphus gestatten die Schlußfolgerung, daß sich der Organisationstypus der Conulata in diesem Polypen in wesentlichen Zügen bis auf den heutigen Tag erhalten hat.

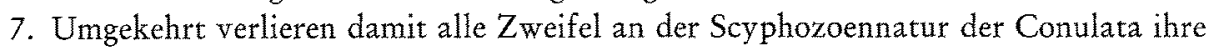
Berechtigung.

8. Auf Grund der Morphologie und Biologie von Stephanoscyphus können wir uns von Bau und Lebensweise der Conulata heute ein besseres Bild machen, als es bis jetzt möglich war. Die bislang vertretene Auffassung, daß sich die erwachsenen Conulata aktiv vom Basalteil ablösten und dann eine vagile oder gar freischwimmende Lebensweise führten, hat nur eine sehr geringe Wahrscheinlichkeit für sich. Die Conulata repräsentierten vielmehr eine reine Polypengeneration.

Der Deutschen Forschungsgemeinschat, die mir die Teilnahme an der "Meteor"-Expedition (1964 bis 1965) in den Indischen Ozean ermöglicht und mich durch Mittel bei der Auswertung des mitgebrachten Lebendmaterials unterstützt hat, möchte ich auch an dieser Stelle herzlich danken. Außerdem bin ich Herrn Prof. Volgt, Hamburg, für die Anregung zu dem Vortrag und für die Hilfe bei der Literaturbeschaffung zu Dank verpflichtet. Mein besonderer Dank gebührt auch meiner bewährten Mitarbeiterin Frau A. REINERS, die die mühevollen Kulturarbeiten mit großer Sorgfalt ausgeführt hat.

\section{ZITIERTE LITERATUR}

Allman, G. J., 1874. On the structure and systematic position of Stephanoscyphus mirabilis, the type of a new order of Hydrozoa. Trans. Linn. Soc. Lond. (Ser. 2: Zool.) 1, 61-66.

BouČEK, B., 1939. Conularida. In: Handbuch der Paläozoologie. Hrsg. von O. H. Schindewolf. Borntraeger, Berlin, 2A (Lfg 5), 113-131.

Brahm, C. \& Geiger, S. R., 1966. Additional record of the scyphozoan Stephanoscyphus simplex Krrkpatrick. Bull. Sth. Calif. Acad. Sci. 65, 47-52.

Chapman, D. M., 1966. Evolution of the scyphistoma. In: The Cnidaria and their evolution. Ed. by W. J. Rees. Acad. pr., Lond. (Symp. zool. Soc. Lond. 16, 51-75.)

Claus, C., 1883. Untersuchungen über die Organisation und Entwicklung der Medusen. Tempsky, Prag; Freytag, Leipzig, 96 pp. 
HaDžI, J., 1963. The evolution of the metazoa. Pergamon pr., Oxford, 499 pp.

Kiderien, H, 1937. Die Conularien, über Bau und Leben der ersten Scyphozoen. Neues /b. Miner. Geol. Paläont. BeilBd. 77B, 113-169.

Knight, J. B., 1937. Conchopeltis Walcott, an Ordovician genus of Conularida. J. Palaeontol. 11, 186-188.

Komá, T., 1935. On Stephanoscypbus and Nausitbö̈. Mem. Coll. Sci. Kyoto Univ. (Ser. B) 10, 289--339.

Kramp, P. L., 1959. Stephanoscyphus (Scyphozoa). Galathea Rep. 1, 173-185.

Leloup, E., 1937. Hydropolypes et Scyphopolypes recueillis par C. Dawydoff sur les côtes de l'Indochine française. Mém. Mus. r. Hist. nat. Belg. (Sér. 2) 12, 1-73.

Lo BIANCO, S. \& MAYER, P., 1890. Spongicola und Nausithoë. Zool. Anz. 13, 687-688.

MetschniKofe, E., 1886. Embryologische Studien an Medusen. Ein Beitrag zur Genealogie der Primitivorgane. Hölder, Wien, 159 pp.

Moore, D. R., 1961. The occurence of Stephanoscypbus corniformis Komal (Scyphozoa) in the western Atlantic. Bull. mar. Sci. Gulf Caribb. 11, 319-320.

Moore, R. C. \& Harrington, H. J., 1956. Scyphozoa. In: Treatise on invertebrate paleontology. Pt F: Coelenterata. Univ. of Kansas pr., Lawrence, Kans., 498 pp. (Repr. 1963)

Mülier, A. H., 1963. Lehrbuch der Paläozoologie. Bd 2: Invertebraten. T. 1: Protozoa. Mollusca (1). 2. Aufl. G. Fischer, Jena, 574 pp.

Naumov, D. W., 1959. Artliche Verschiedenheiten der Polypengeneration der Coronatae. [Russ.] Dokl. Akad. Nauk SSSR 126, 902-904.

Richter, R. \& Richter, E., 1930. Bemerkenswert erhaltene Conularien und ihre Gattungsgenossen im Hunsrückschiefer (Unterdevon) des Rheinlandes. Senckenbergiana 12, 152-171.

Schmidt, W. \& Terchmülier, M., 1958, Neue Funde von Sphenothallus auf dem westeuropäischen Festland, insbesondere in Belgien, und ergänzende Beobachtungen zur Gattung Sphenothallus. Publs Ass. Etude Paléont. Stratigr. howill. 33, 1-34.

Termier, G. \& Termier, H., 1948. Position systématique et biologie des Conulaires. Revue scient., Paris 86, 711-722.

Thiel, HJ., 1966. The evolution of Scyphozoa. A review. In: The Cnidaria and their evolution. Ed. by W. J. Rees. Acad. pr., Lond. (Symp. zool. Soc. Lond. 16, 77-117.)

ThIEl, M. E., 1936. Coronatae. Bronn's Kl. Ordng. Tierreich Bd 2 (Abt. 2), Lfg 3, 321-480.

Vinogradow, A. P., 1953. The elementary chemical composition of marine organisms. Mem. Sears Fdn mar. Res, 2, 1-647.

Werner, B., 1967. Morphologie, Systematik und Lebensgeschichte von Stephanoscyphus (Scyphozoa Coronatae), sowie seine Bedeutung für die Evolution der Scyphozoa. In: Verh. dt. zool. Ges. Göttingen 1966 [Zool. Anz. (Suppl. Bd) 30] (im Druck). 